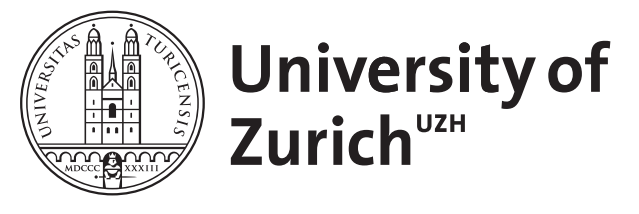

\title{
Results on normal forms for FPU chains
}

\author{
Henrici, A ; Kappeler, T
}

\begin{abstract}
In this paper we prove, among other results, that near the equilibirum position, any periodic FPU chain with an odd number $\mathrm{N}$ of particles admits a Birkhoff normal form up to order 4, whereas any periodic FPU chain with $\mathrm{N}$ even admits a resonant normal form up to order 4 . This resonant normal form of order 4 turns out to be completely integrable. Further, for N odd, we obtain an explicit formula of the Hessian of its Hamiltonian at the fixed point.
\end{abstract}

DOI: https://doi.org/10.1007/s00220-007-0387-z

Posted at the Zurich Open Repository and Archive, University of Zurich ZORA URL: https://doi.org/10.5167/uzh-7209

Journal Article

Published Version

Originally published at:

Henrici, A; Kappeler, T (2008). Results on normal forms for FPU chains. Communications in Mathematical Physics, 278(1):145-177.

DOI: https://doi.org/10.1007/s00220-007-0387-z 


\title{
Results on Normal Forms for FPU Chains
}

\author{
Andreas Henrici ${ }^{\star}$, Thomas Kappeler ${ }^{\star \star}$ \\ Institut für Mathematik, Universität Zürich, Winterthurerstrasse 190, CH-8057 Zürich, Switzerland. \\ E-mail: tk@math.unizh.ch; andreas.henrici@math.unizh.ch
}

Received: 16 February 2007 / Accepted: 19 May 2007

Published online: 22 November 2007 - C Springer-Verlag 2007

\begin{abstract}
In this paper we prove, among other results, that near the equilibirum position, any periodic FPU chain with an odd number $N$ of particles admits a Birkhoff normal form up to order 4, whereas any periodic FPU chain with $N$ even admits a resonant normal form up to order 4 . This resonant normal form of order 4 turns out to be completely integrable. Further, for $N$ odd, we obtain an explicit formula of the Hessian of its Hamiltonian at the fixed point.
\end{abstract}

\section{Introduction}

In this paper we consider FPU chains with $N$ particles of equal mass, normalized to be one. Such chains have been introduced by Fermi, Pasta, and Ulam [5], as models to test numerically the principle of thermalization as $N$ gets larger and larger. A FPU chain consists of a string of particles moving on the line or the circle interacting only with their nearest neighbors through nonlinear springs. Its Hamiltonian is given by

$$
H_{V}=\frac{1}{2} \sum_{n=1}^{N} p_{n}^{2}+\sum_{n=1}^{N} V\left(q_{n}-q_{n+1}\right)
$$

where $V: \mathbb{R} \rightarrow \mathbb{R}$ is a smooth potential. The corresponding Hamiltonian equations read $(1 \leq n \leq N)$

$$
\begin{aligned}
& \dot{q}_{n}=\partial_{p_{n}} H_{V}=p_{n}, \\
& \dot{p}_{n}=-\partial_{q_{n}} H_{V}=-V^{\prime}\left(q_{n}-q_{n+1}\right)+V^{\prime}\left(q_{n-1}-q_{n}\right) .
\end{aligned}
$$

* Supported in part by the Swiss National Science Foundation.

$\star \star$ Supported in part by the Swiss National Science Foundation, the programme SPECT and the European Community through the FP6 Marie Curie RTN ENIGMA (MRTN-CT-2004-5652). 
Here $q_{n}$ denotes the displacement of the $n^{\text {th }}$ particle from its equilibrium position and $p_{n}$ is its momentum. If not stated otherwise we assume periodic boundary conditions

$$
\left(q_{i+N}, p_{i+N}\right)=\left(q_{i}, p_{i}\right) \quad \forall i \in\{0,1\} .
$$

Without loss of generality, the potential $V: \mathbb{R} \rightarrow \mathbb{R}$ is assumed to have a Taylor expansion at 0 of the form

$$
V(x)=\kappa\left(\frac{1}{2} x^{2}-\frac{\alpha}{3 !} x^{3}+\frac{\beta}{4 !} x^{4}+\ldots\right),
$$

where $\kappa$ is the (linear) spring constant normalized to be 1 and $\alpha, \beta \in \mathbb{R}$ are parameters measuring the strength of the nonlinear interaction. The minus sign in front of the parameter $\alpha$ in the expansion (2) turns out to be convenient for later computations. Substituting the expression (2) for $V$ into (1), the corresponding expansion of $H_{V}$ is given by

$$
H_{V}=\frac{1}{2} \sum_{n=1}^{N} p_{n}^{2}+\frac{1}{2} \sum_{n=1}^{N}\left(q_{n+1}-q_{n}\right)^{2}+\frac{\alpha}{3 !} \sum_{n=1}^{N}\left(q_{n+1}-q_{n}\right)^{3}+\frac{\beta}{4 !} \sum_{n=1}^{N}\left(q_{n+1}-q_{n}\right)^{4}+\ldots
$$

For any FPU chain, the total momentum $P=\frac{1}{N} \sum_{n=1}^{N} p_{n}$ is an integral of motion, and therefore the center of mass $Q=\frac{1}{N} \sum_{n=1}^{N} q_{n}$ evolves with constant velocity. Hence any FPU chain can be viewed as a family of Hamiltonian systems of $N-1$ degrees of freedom, parametrized by the vector of initial conditions $(Q, P) \in \mathbb{R}^{2}$ with Hamiltonian independent of $Q$. In particular, for $N=2$ any FPU chain is integrable, and hence we will concentrate on the case $N \geq 3$. Further note that for any vector $(Q, P) \in \mathbb{R}^{2}$, the origin in $\mathbb{R}^{2 N-2}$ is an equilibrium point of the corresponding system. The momentum of such an equilibrium point is given by the constant vector $\left(p_{1}, \ldots, p_{N}\right)=P(1, \ldots, 1)$.

Introduce the function $I=\left(I_{k}\right)_{1 \leq k \leq N-1}$, defined on $\mathbb{R}^{2 N-2}$ with values in $\mathbb{R}^{N-1}$,

$$
I_{k}=\frac{1}{2}\left(x_{k}^{2}+y_{k}^{2}\right)
$$

Further define the function $H_{\alpha, \beta}: \mathbb{R}^{N-1} \rightarrow \mathbb{R}$, given by

$$
H_{\alpha, \beta}(I):=2 \sum_{k=1}^{N-1} \sin \frac{k \pi}{N} I_{k}+\frac{1}{4 N} \sum_{k=1}^{N-1} c_{k} I_{k}^{2}+\frac{\beta-\alpha^{2}}{2 N} \sum_{\substack{l \neq m \\ 1 \leq l, m \leq N-1}} \sin \frac{l \pi}{N} \sin \frac{m \pi}{N} I_{l} I_{m},
$$

where $c_{k} \equiv c_{k}(\alpha, \beta):=\alpha^{2}+\left(\beta-\alpha^{2}\right) \sin ^{2} \frac{k \pi}{N}$.

Surprisingly, the properties of periodic FPU chains near the equilibrium point strongly depend on the parity of the number $N$ of particles. If $N$ is odd, our results are the following ones:

Theorem 1.1. Let $\alpha, \beta \in \mathbb{R}$ with $(\alpha, \beta) \neq(0,0)$. If $N \geq 3$ is odd, then any periodic FPU chain admits a Birkhoff normal form of order 4 . More precisely, there are canonical coordinates $\left(x_{k}, y_{k}\right)_{1 \leq k \leq N-1}$ so that the Hamiltonian of any FPU chain, when expressed in these coordinates, takes the form

$$
\frac{N P^{2}}{2}+H_{\alpha, \beta}(I)+O\left(|(x, y)|^{5}\right)
$$

with $H_{\alpha, \beta}(I)$ given by (5). 
Corollary 1.2. Near the equilibrium state, any FPU chain with an odd number $N$ of particles can be approximated up to order 4 relative to its center of mass coordinates by an integrable system of $N-1$ harmonic oscillators which are coupled at fourth order except if $\beta=\alpha^{2}$ (Toda case).

Denote by $Q_{\alpha, \beta}$ the Hessian of $H_{\alpha, \beta}(I)$ at $I=0$. Note that $Q_{\alpha, \beta}$ is an $(N-1) \times(N-1)$ matrix which only depends on the parameters $\alpha$ and $\beta$. For the following result we do not have to assume that $N$ is odd.

Theorem 1.3. (i) For any given $\alpha \in \mathbb{R} \backslash\{0\}$, $\operatorname{det}\left(Q_{\alpha, \beta}\right)$ is a polynomial in $\beta$ of degree $N-1$ and has $N-1$ real zeroes (counted with multiplicities). When listed in increasing order, the zeroes $\beta_{k}=\beta_{k}(\alpha)$ satisfy

$$
0<\beta_{1}<\alpha^{2}, \quad 2 \alpha^{2}<\beta_{2} \leq \ldots \leq \beta_{N-1}
$$

and contain the $\left\llcorner\frac{N-1}{2}\right\lrcorner$ distinct numbers

$$
\alpha^{2}\left(1+\left(\sin ^{2} \frac{k \pi}{N}\right)^{-1}\right) \quad\left(1 \leq k \leq\left\llcorner\frac{N-1}{2}\right\lrcorner\right) .
$$

Moreover index $\left(Q_{\alpha, \beta}\right)$, defined as the number of negative eigenvalues of $Q_{\alpha, \beta}$, is given by

$$
\operatorname{index}\left(Q_{\alpha, \beta}\right)= \begin{cases}1 & \text { for } \beta<\beta_{1} \\ 0 & \text { for } \beta_{1}<\beta<\beta_{2} \\ N-2 & \text { for } \beta>\beta_{N-1}\end{cases}
$$

(ii) For $\alpha=0, \operatorname{det}\left(Q_{0, \beta}\right)$ is a polynomial in $\beta$ of degree $N-1$, and $\beta=0$ is the only zero of $\operatorname{det}\left(Q_{0, \beta}\right)$. It has multiplicity $N-1$, and the index of $Q_{0, \beta}$ is given by

$$
\operatorname{index}\left(Q_{0, \beta}\right)= \begin{cases}1 & \text { for } \beta<0, \\ N-2 & \text { for } \beta>0 .\end{cases}
$$

Periodic FPU chains with an even number $N$ of particles do not admit a Birkhoff normal form up to order 4 due to resonances except if $\beta=\alpha^{2}$ (Toda case). Our analysis of odd FPU chains leads in the case of even FPU chains to a resonant Birkhoff normal form up to order 4.

Define the functions $J=\left(J_{k}\right)_{1 \leq k \leq N-1}$ and $M=\left(M_{k}\right)_{1 \leq k \leq N-1}$ on $\mathbb{R}^{2 N-2}$ with values in $\mathbb{R}^{N-1}$ given by

$$
J_{k}=\frac{1}{2}\left(x_{k} x_{N-k}+y_{k} y_{N-k}\right) \text { and } M_{k}=\frac{1}{2}\left(x_{k} y_{N-k}-x_{N-k} y_{k}\right),
$$

and introduce

$$
R_{\alpha, \beta}(J, M):=\frac{\beta-\alpha^{2}}{4 N}\left(R(J, M)+R_{\frac{N}{4}}(J, M)\right),
$$

where

$$
R(J, M)=4 \sum_{1 \leq k<\frac{N}{4}} \sin \frac{2 k \pi}{N}\left(J_{k} J_{\frac{N}{2}-k}-M_{k} M_{\frac{N}{2}-k}\right)
$$


and

$$
R_{\frac{N}{4}}(J, M)= \begin{cases}J_{\frac{N}{4}}^{2}-M_{\frac{N}{4}}^{2} & \text { if } \frac{N}{4} \in \mathbb{N} \\ 0 & \text { otherwise. }\end{cases}
$$

Note that for $\alpha, \beta \in \mathbb{R}$ with $\beta=\alpha^{2}$ (Toda case), the expression $R_{\alpha, \beta}$ vanishes.

Our main results on even periodic FPU chains are the following ones:

Theorem 1.4. Let $\alpha, \beta \in \mathbb{R}$ with $(\alpha, \beta) \neq(0,0)$. If $N \geq 4$ is even, there are canonical coordinates $\left(x_{k}, y_{k}\right)_{1 \leq k \leq N-1}$ so that the Hamiltonian of any FPU chain, when expressed in these coordinates, takes the form $G_{\alpha, \beta}(I, J, M)+O\left(|(x, y)|^{5}\right)$, where

$$
G_{\alpha, \beta}(I, J, M)=\frac{N P^{2}}{2}+H_{\alpha, \beta}(I)-R_{\alpha, \beta}(J, M),
$$

and where $H_{\alpha, \beta}(I)$ and $R_{\alpha, \beta}(J, M)$ are given by (5) and (7), respectively.

It is quite remarkable that $G_{\alpha, \beta}$ turns out to be completely integrable. To make this statement more precise, introduce for $1 \leq k<\frac{N}{4}$,

$$
\begin{aligned}
K_{k, \alpha, \beta}(J, M)= & d_{k}^{-}\left(J_{k}^{2}+M_{k}^{2}\right)+d_{\frac{N}{2}-k}^{-}\left(J_{\frac{N}{2}-k}^{2}+M_{\frac{N}{2}-k}^{2}\right) \\
& -2\left(\beta-\alpha^{2}\right) \sin \frac{2 k \pi}{N}\left(J_{k} J_{\frac{N}{2}-k}-M_{k} M_{\frac{N}{2}-k}\right),
\end{aligned}
$$

where $d_{k}^{-}:=-\alpha^{2}+\left(\beta-\alpha^{2}\right) \sin ^{2} \frac{k \pi}{N}$, and let

$$
K_{\frac{N}{4}, \alpha, \beta}(J, M)= \begin{cases}-\alpha^{2} J_{\frac{N}{4}}^{2}+\left(\beta-2 \alpha^{2}\right) M_{\frac{N}{4}}^{2} & \text { if } N \equiv 0 \bmod 4 \\ 0 & \text { otherwise. }\end{cases}
$$

Theorem 1.5. Let $N \geq 4$ be an even integer. Then for any $\alpha, \beta \in \mathbb{R}$ with $(\alpha, \beta) \neq(0,0)$, the truncated FPU Hamiltonian $G_{\alpha, \beta}$ given by (8) is completely integrable. The following $N-1$ quantities are functionally independent integrals in involution:

$$
\left(I_{k}+I_{N-k}\right)_{1 \leq k \leq \frac{N}{2}}, \quad\left(I_{k}+I_{\frac{N}{2}+k}\right)_{1 \leq k<\frac{N}{4}}, \quad\left(K_{k, \alpha, \beta}\right)_{1 \leq k \leq \frac{N}{4}} .
$$

Application 1. In the case where $N$ is odd, Theorems 1.1 and 1.3 allow to apply for any given $\alpha \in \mathbb{R}$ the classical KAM theorem (see e.g. [11]) near the equilibrium point to the FPU chain with Hamiltonian $H_{V}$ for a real analytic potential $V(x)=\frac{1}{2} x^{2}-\frac{\alpha}{3 !} x^{3}+$ $\frac{\beta}{4 !} x^{4}+\ldots$ with $\beta \in \mathbb{R} \backslash\left\{\beta_{1}(\alpha), \ldots, \beta_{N-1}(\alpha)\right\}$. Moreover, note that for any given $\alpha \in \mathbb{R} \backslash\{0\}$, the Hessian $Q_{\alpha, \beta}$ of $H_{\alpha, \beta}$ is positive definite for any $\beta$ satisfying $\beta_{1}(\alpha)<$ $\beta<\beta_{2}(\alpha)$, in particular for $\alpha^{2} \leq \beta \leq 2 \alpha^{2}$. Hence one can apply Nekhoroshev's theorem (see e.g. [12]) near the equilibrium point to the FPU chain with Hamiltonian $H_{V}$ for $V$ with such $\beta$ 's. These perturbation results confirm long standing conjectures see e.g. [3]. 
Application 2. Theorem 1.5 can be used to show that any FPU chain with Dirichlet boundary conditions admits a Birkhoff normal form up to order 4 near the equilibrium point $(q, p)=(0,0)$ by viewing such a system as an invariant symplectic submanifold of a periodic FPU chain. Consider a chain with $N^{\prime}\left(N^{\prime} \geq 3\right.$, not necessarily even) moving particles, Hamiltonian

$$
H_{V}^{D}=\frac{1}{2} \sum_{n=1}^{N^{\prime}} p_{n}^{2}+\sum_{n=1}^{N^{\prime}} V\left(q_{n}-q_{n+1}\right)
$$

and with endpoints assumed to be fixed, $q_{0}=q_{N^{\prime}+1}=0$.

Theorem 1.6. Let $\alpha, \beta \in \mathbb{R}$ with $(\alpha, \beta) \neq(0,0)$. Then any FPU chain with $N^{\prime} \geq 3$ moving particles and satisfying Dirichlet boundary conditions admits a Birkhoff normal form of order 4 , i.e. there are canonical coordinates $\left(x_{k}, y_{k}\right)_{1 \leq k \leq N^{\prime}}$ so that $H_{V}^{D}$ takes the form

$$
\frac{\left(N^{\prime}+1\right) P^{2}}{2}+H_{\alpha, \beta}^{D}(I)+O\left(|(x, y)|^{5}\right),
$$

where $I=\left(I_{1}, \ldots, I_{N^{\prime}}\right)$ and $H_{\alpha, \beta}^{D}(I)$ is given by

$$
\begin{aligned}
& 2 \sum_{k=1}^{N^{\prime}} s_{k} I_{k}+\frac{1}{16\left(N^{\prime}+1\right)} \sum_{k=1}^{N^{\prime}}\left(\alpha^{2}+3\left(\beta-\alpha^{2}\right) s_{k}^{2}\right) I_{k}^{2} \underbrace{+\frac{\beta-\alpha^{2}}{32\left(N^{\prime}+1\right)} I_{\frac{N}{4}}^{2}}_{\text {only if } \frac{N}{4} \in \mathbb{N}} \\
& +\frac{\beta-\alpha^{2}}{16\left(N^{\prime}+1\right)}\left(\sum_{\substack{l \neq m \\
1 \leq l, m \leq N^{\prime}}} 4 s_{l} s_{m} I_{l} I_{m}-\sum_{k=1}^{N^{\prime}} s_{2 k} I_{k} I_{N^{\prime}+1-k}\right),
\end{aligned}
$$

with $s_{k}=\sin \frac{k \pi}{2 N^{\prime}+2}\left(1 \leq k \leq N^{\prime}\right)$.

Similarly as in the case of odd periodic FPU chains, one can analyze the Hessian $Q_{\alpha, \beta}^{D}$ of $H_{\alpha, \beta}^{D}(I)$ at $I=0$.

Theorem 1.7. (i) For any given $\alpha \in \mathbb{R} \backslash\{0\}$, $\operatorname{det}\left(Q_{\alpha, \beta}^{D}\right)$ is a polynomial in $\beta$ of degree $N^{\prime}$ and has $N^{\prime}$ real zeroes (counted with multiplicities). When listed in increasing order, the zeroes $\beta_{k}=\beta_{k}(\alpha)$ satisfy

$$
\beta_{1} \leq \ldots \leq \beta_{\left\ulcorner\frac{N^{\prime}+1}{2}\right\urcorner}<\alpha^{2}<\beta_{\left\ulcorner\frac{N^{\prime}+3}{2}\right\urcorner} \leq \ldots \leq \beta_{N^{\prime}}
$$

Moreover index $\left(Q_{\alpha, \beta}^{D}\right)$, defined as the number of negative eigenvalues of $Q_{\alpha, \beta}^{D}$, is given by

$$
\operatorname{index}\left(Q_{\alpha, \beta}^{D}\right)= \begin{cases}\left\ulcorner\frac{N^{\prime}+1}{2}\right\urcorner & \text { for } \beta<\beta_{1} \\ 0 & \text { for } \beta_{\left\ulcorner\frac{N^{\prime}+1}{2}\right\urcorner}<\beta<\beta_{\left\ulcorner\frac{N^{\prime}+3}{2}\right\urcorner .} . \\ \left\llcorner\frac{N^{\prime}-1}{2}\right\lrcorner & \text { for } \beta>\beta_{N^{\prime}}\end{cases}
$$


(ii) For $\alpha=0, \operatorname{det}\left(Q_{0, \beta}^{D}\right)$ is a polynomial in $\beta$ homogeneous of degree $N^{\prime}$. Hence $\beta=0$ is the only zero of $\operatorname{det}\left(Q_{0, \beta}^{D}\right)$ and the index of $Q_{0, \beta}^{D}$ is given by

$$
\operatorname{index}\left(Q_{0, \beta}^{D}\right)=\left\{\begin{array}{ll}
\left\ulcorner\frac{N^{\prime}+1}{2}\right\urcorner & \text { for } \beta<0 \\
\left\llcorner\frac{N^{\prime}-1}{2}\right\lrcorner & \text { for } \beta>0
\end{array}\right. \text {. }
$$

Related work. Theorem 1.1 and Theorem 1.3 improve on earlier results of Rink [13]. In particular, Theorem 1.3 treats the case $\alpha \neq 0$ which was described in [13] as one of the major open problems in this topic. In fact, Theorem 1.1 and Theorem 1.3 solve all open problems stated in [13] for $N$ odd. Theorems $1.5,1.6$, and 1.7 considerably improve on earlier results of Rink in [13,14], and [15] (cf. also [10]) where the case $\alpha=0$ was treated. Our approach has been shaped by our earlier work on the Toda lattice [6,7]. The latter one, introduced by Toda [16] and extensively studied in the sequel, is a special FPU chain which is completely integrable. It turns out that the canonical transformations which near the equilibrium bring the Toda lattice into Birkhoff normal form can be used for any FPU chain. In other words, the existence of the Birkhoff normal form stated in Theorem 1.1 is, at least partially, a consequence of the fact that the family of FPU chains, parametrized by $\alpha, \beta, \ldots$, contains an integrable system, namely the Toda lattice.

The literature on FPU chains is huge - see e.g. the recent survey article [3] by Berman and Izrailev. One of the most important open problems in this topic is the investigation of the dynamics of FPU chains when the number of particles gets larger and larger. It is likely that our results combined with results for the $\mathrm{KdV}$ equation established in [9] can be used for this purpose. For recent contributions in this direction see e.g. [1,2].

Outline. In Sect. 2, we review the notion of Birkhoff normal form. We show Theorem 1.1 in Sects. 3 and 4 and Theorem 1.4 in Sect. 5, whereas Theorem 1.3 will be proved in Sect. 6. Theorems 1.5, 1.6, and 1.7 together with a detailed analysis of the (quite complicated) level sets of the integrals in Theorem 1.5 are proved in the subsequent paper [8].

\section{Birkhoff Normal Form}

Consider an isolated equilibrium of a Hamiltonian system on some $2 n$-dimensional symplectic manifold, i.e. an isolated singular point of the Hamiltonian vector field. Neglecting an irrelevant additive constant, the Hamiltonian, when expressed in canonical coordinates $w=(q, p)$ near the equilibrium with coordinates $q=0, p=0$, then has the form

$$
H=\frac{1}{2}\langle A w, w\rangle+\ldots,
$$

where $A$ is the symmetric $2 n \times 2 n$-Hessian of $H$ at 0 and the dots stand for terms of higher order in $w$. We now assume that the equilibrium point $w=0$ is elliptic, i.e. the spectrum of the linearized system, $\dot{w}=J A w$, is purely imaginary, $\operatorname{spec}(J A)=$ $\left\{ \pm i \lambda_{1}, \ldots, \pm i \lambda_{n}\right\}$ with real numbers $\lambda_{1}, \ldots, \lambda_{n}$. Here $J=\left(\begin{array}{cc}0 & I d_{n} \\ -I d_{n} & 0\end{array}\right)$ is the standard symplectic structure of $\mathbb{R}^{2 n}$. If $\operatorname{spec}(J A)$ is simple there exists a linear symplectic 
change of coordinates which brings the quadratic part of the Hamiltonian into normal form. Denoting the new coordinates by the same symbols as the old ones one has

$$
\langle A w, w\rangle=\sum_{i=1}^{n} \lambda_{i}\left(q_{i}^{2}+p_{i}^{2}\right)
$$

Definition 2.1. A Hamiltonian $H$ is in Birkhoff normal form up to order $m \geq 2$, if it is of the form

$$
H=N_{2}+N_{4}+\cdots+N_{m}+H_{m+1}+\cdots,
$$

where the $N_{k}, 2 \leq k \leq m$, are homogeneous polynomials of order $k$, which are actually functions of $q_{1}^{2}+p_{1}^{2}, \ldots, q_{n}^{2}+p_{n}^{2}$, and where $H_{m+1}+\ldots$ stands for (arbitrary) terms of order strictly greater than $m$. If this holds for any $m$, the Hamiltonian is said to be in Birkhoff normal form and the coordinates $\left(q_{i}, p_{i}\right)_{1 \leq i \leq n}$ are referred to as Birkhoff coordinates.

Note that if a Hamiltonian $H$ admits a Birkhoff normal form of order $m$, the coefficients of the expansion (10) up to order $m$ are uniquely determined, as long as the normalizing transformation is of the form id $+\ldots$. However, the normalizing transformation is by no means unique.

There are well known theorems guaranteeing the existence of a Birkhoff normal form up to order $m$ assuming that the frequencies $\lambda_{1}, \ldots, \lambda_{n}$ satisfy certain nonresonance conditions - see e.g. Theorem 4.3 in [9]. However, in the case under investigation, the nonresonance conditions for $m=4$ are not satisfied. We will show by an explicit computation that an arbitrary odd periodic FPU chain admits a Birkhoff normal form up to order 4, while an even periodic FPU chain only admits a resonant normal form except in the case $\beta=\alpha^{2}$ (Toda case).

\section{Birkhoff Normal Form up to Order 2}

Following the procedure of constructing Birkhoff coordinates for the Toda lattice [6] we first introduce relative coordinates (which are a canonical version of the Flaschka coordinates),

$$
v_{i}:=q_{i+1}-q_{i}(1 \leq i \leq N-1) \text { and } \quad v_{N}:=\frac{1}{N} \sum_{i=1}^{N} q_{i}
$$

and denote by $\left(u_{i}\right)_{1 \leq i \leq N}$ the corresponding conjugate variables. It turns out that $u_{N}=$ $N \cdot P=\sum_{i=1}^{N} p_{i}$ and $q_{N+1}-q_{N}=-\sum_{k=1}^{N-1} v_{k}$. The Hamiltonian $H_{V}$ in (3), when expressed in these coordinates, takes the form $H_{V}=\frac{N P^{2}}{2}+\tilde{H}_{V}$ with $\tilde{H}_{V}=H_{u}+H_{v}$, where $H_{u}$ and $H_{v}$ only depend on $u=\left(u_{i}\right)_{1 \leq i \leq N-1}$ and $v=\left(v_{i}\right)_{1 \leq i \leq N-1}$, respectively, 
and are given by

$$
\begin{aligned}
H_{u}= & \frac{1}{2}\left(u_{1}^{2}+\sum_{l=1}^{N-2}\left(u_{l+1}-u_{l}\right)^{2}+u_{N-1}^{2}\right) \\
H_{v}= & \frac{1}{2}\left(\sum_{k=1}^{N-1} v_{k}^{2}+\left(\sum_{k=1}^{N-1} v_{k}\right)^{2}\right)+\frac{\alpha}{3 !}\left(\sum_{k=1}^{N-1} v_{k}^{3}-\left(\sum_{k=1}^{N-1} v_{k}\right)^{3}\right) \\
& +\frac{\beta}{4 !}\left(\sum_{k=1}^{N-1} v_{k}^{4}+\left(\sum_{k=1}^{N-1} v_{k}\right)^{4}\right)+O\left(v^{5}\right) .
\end{aligned}
$$

To bring $\tilde{H}_{V}=H_{u}+H_{v}$ into Birkhoff normal form up to order 2 we introduce new coordinates $\left(\xi_{k}, \eta_{k}\right)_{1 \leq k \leq N-1}$. It turns out to be convenient to use complex notation, i.e. for $1 \leq k \leq N-1$,

$$
\zeta_{k}:=\frac{1}{\sqrt{2}}\left(\xi_{k}-i \eta_{k}\right), \quad \zeta_{-k}:=\frac{1}{\sqrt{2}}\left(\xi_{k}+i \eta_{k}\right),
$$

where the minus sign in the definition of $\zeta_{k}$ is chosen so that $d \zeta_{k} \wedge d \zeta_{-k}=i d \xi_{k} \wedge d \eta_{k}$. The vector $\zeta=\left(\zeta_{k}\right)_{1 \leq|k| \leq N-1}$ is an element in the space

$$
Z:=\left\{z=\left(z_{k}\right)_{1 \leq|k| \leq N-1} \in \mathbb{C}^{2 N-2}: z_{-k}=\overline{z_{k}} \quad \forall 1 \leq k \leq N-1\right\} .
$$

Further introduce the notation

$$
\lambda_{k}:=\left|\sin \frac{k \pi}{N}\right|^{\frac{1}{2}} \quad(0 \leq|k| \leq N-1)
$$

The proposed linear transformation $Z \rightarrow \mathbb{R}^{2 N-2}, \zeta \mapsto(v, u)$ is then defined by

$$
\begin{aligned}
u_{1}(\zeta) & =\frac{1}{\sqrt{N}} \sum_{1 \leq|k| \leq N-1} \lambda_{k} \zeta_{k} \\
u_{l+1}(\zeta)-u_{l}(\zeta) & =\frac{1}{\sqrt{N}} \sum_{1 \leq|k| \leq N-1} \lambda_{k} e^{2 \pi i l k / N} \zeta_{k} \quad(1 \leq l \leq N-2) \\
-u_{N-1}(\zeta) & =\frac{1}{\sqrt{N}} \sum_{1 \leq|k| \leq N-1} \lambda_{k} e^{2 \pi i(N-1) k / N} \zeta_{k}
\end{aligned}
$$

and

$$
v_{l}(\zeta)=\frac{1}{\sqrt{N}} \sum_{1 \leq|k| \leq N-1} \lambda_{k} e^{2 \pi i l k / N} e^{-i \pi k / N} \zeta_{k} \quad(1 \leq l \leq N-1)
$$

Note that (16) is actually a consequence of (14) and (15). As explained in [7] it follows from the construction of the Birkhoff map of the Toda lattice that this map is a canonical isomorphism - see Lemma B.1 in Appendix B for a self-contained proof. 
When expressed in the new coordinates, $H_{u}$ and $H_{v}$ take the form

$$
\begin{aligned}
& H_{u}(\zeta)=\frac{1}{2} G_{2}(\zeta) \\
& H_{v}(\zeta)=\frac{1}{2} G_{2}(\zeta)+\alpha G_{3}(\zeta)+\beta G_{4}(\zeta)+O\left(\zeta^{5}\right)
\end{aligned}
$$

where

$$
\begin{aligned}
G_{2} & :=2 \sum_{k=1}^{N-1} \lambda_{k}^{2} \zeta_{k} \zeta_{-k}, \\
G_{3} & :=\frac{1}{6 \sqrt{N}} \sum_{\left(k, k^{\prime}, k^{\prime \prime}\right) \in K_{3}}(-1)^{\left(k+k^{\prime}+k^{\prime \prime}\right) / N} \lambda_{k} \lambda_{k^{\prime}} \lambda_{k^{\prime \prime}} \zeta_{k} \zeta_{k^{\prime}} \zeta_{k^{\prime \prime}}, \\
G_{4} & :=\frac{1}{24 N} \sum_{\left(k, k^{\prime}, k^{\prime \prime}, k^{\prime \prime \prime}\right) \in K_{4}}(-1)^{\left(k+k^{\prime}+k^{\prime \prime}+k^{\prime \prime \prime}\right) / N} \lambda_{k} \lambda_{k^{\prime}} \lambda_{k^{\prime \prime}} \lambda_{k^{\prime \prime \prime}} \zeta_{k} \zeta_{k^{\prime}} \zeta_{k^{\prime \prime}} \zeta_{k^{\prime \prime \prime}},
\end{aligned}
$$

with

$$
\begin{aligned}
K_{3}:= & \left\{\left(k, k^{\prime}, k^{\prime \prime}\right) \in \mathbb{Z}^{3}: 1 \leq|k|,\left|k^{\prime}\right|,\left|k^{\prime \prime}\right| \leq N-1\right. \\
& \text { and } \left.k+k^{\prime}+k^{\prime \prime} \equiv 0 \bmod N\right\}
\end{aligned}
$$

and

$$
\begin{aligned}
K_{4}:= & \left\{\left(k, k^{\prime}, k^{\prime \prime}, k^{\prime \prime \prime}\right) \in \mathbb{Z}^{4}: 1 \leq|k|,\left|k^{\prime}\right|,\left|k^{\prime \prime}\right|,\left|k^{\prime \prime \prime}\right| \leq N-1\right. \\
& \text { and } \left.k+k^{\prime}+k^{\prime \prime}+k^{\prime \prime \prime} \equiv 0 \bmod N\right\} .
\end{aligned}
$$

Note that $G_{2}, G_{3}$, and $G_{4}$ are independent of $\alpha$ and $\beta$. In particular they came up in [7] when we computed the Birkhoff normal form of the Toda lattice. For a detailed derivation of the formulas for $G_{2}, G_{3}$, and $G_{4}$ see Appendix B. Summarizing the results of this section we have that

$$
\tilde{H}_{V}(\zeta)=G_{2}(\zeta)+\alpha G_{3}(\zeta)+\beta G_{4}(\zeta)+O\left(\zeta^{5}\right)
$$

is in Birkhoff normal form up to order 2. As a consequence, $\zeta=0$ is an elliptic fixed point of the Hamiltonian $\tilde{H}_{V}$.

\section{Proof of Theorem 1.1.}

We now begin by transforming $\tilde{H}_{V}(\zeta)$ into its Birkhoff normal form up to order 4 . Here we follow a standard procedure - see e.g. Sect. 14 in [9]. The phase space $Z$, defined in (13), is endowed with the Poisson bracket

$$
\{F, G\}=i \sum_{1 \leq|k| \leq N-1} \sigma_{k} \frac{\partial F}{\partial \zeta_{k}} \frac{\partial G}{\partial \zeta_{-k}},
$$

where $\sigma_{k}=\operatorname{sgn}(k)$ is the sign of $k$. The Hamiltonian vector field $X_{F}$ associated to the Hamiltonian $F$ is then given by $X_{F}=i \sum_{1 \leq|k| \leq N-1} \sigma_{k} \frac{\partial F}{\partial \zeta_{-k}} \frac{\partial}{\partial \zeta_{k}}$. With a first canonical 
transformation we want to eliminate the third order term $\alpha G_{3}$ in $\tilde{H}_{V}(\zeta)$. By a by now standard precedure we construct such a canonical transformation on the phase space $z$ as the time-1-map $\Psi_{1}:=\left.X_{\alpha F_{3}}^{t}\right|_{t=1}$ of the flow $X_{\alpha F_{3}}^{t}$ of a real analytic Hamiltonian $\alpha F_{3}$ which is a homogeneous polynomial in $\zeta_{k}(1 \leq|k| \leq N-1)$ of degree 3 and solves the homological equation

$$
\left\{G_{2}, \alpha F_{3}\right\}+\alpha G_{3}=0 .
$$

To simplify notation we momentarily write $F$ instead of $\alpha F_{3}$ and $H$ instead of $\tilde{H}_{V}$. Assuming for the moment that (23) can be solved and that $X_{F}^{t}$ is defined for $0 \leq t \leq 1$ in some neighbourhood of the origin in $Z$, we can use Taylor's formula to expand $H \circ X_{F}^{t}$ around $t=0$,

$$
\begin{aligned}
H \circ X_{F}^{t} & =H \circ X_{F}^{0}+\int_{0}^{t} \frac{d}{d s}\left(H \circ X_{F}^{s}\right) d s \\
& =H+\int_{0}^{t}\{H, F\} \circ X_{F}^{s} d s \\
& =H+t\{H, F\}+\int_{0}^{t} d s \int_{0}^{s} d s^{\prime} \frac{d}{d s^{\prime}}\left(\{H, F\} \circ X_{F}^{s^{\prime}}\right) \\
& =H+t\{H, F\}+\int_{0}^{t}(t-s)\{\{H, F\}, F\} \circ X_{F}^{s} d s .
\end{aligned}
$$

When evaluating this expression at $t=1$, one gets

$$
\begin{aligned}
H \circ \Psi_{1}= & G_{2}+\left\{G_{2}, F\right\}+\int_{0}^{1}(1-t)\left\{\left\{G_{2}, F\right\}, F\right\} \circ X_{F}^{t} d t \\
& +\alpha G_{3}+\int_{0}^{1}\left\{\alpha G_{3}, F\right\} \circ X_{F}^{t} d t+\beta G_{4}+O\left(\zeta^{5}\right) .
\end{aligned}
$$

Using that $\left\{G_{2}, F\right\}+\alpha G_{3}=0$, the latter expression simplifies and we get

$$
H \circ \Psi_{1}=G_{2}+\int_{0}^{1} t\left\{\alpha G_{3}, F\right\} \circ X_{F}^{t} d t+\beta G_{4}+O\left(\zeta^{5}\right) .
$$

Integrating by parts once more and taking into account that $F \equiv \alpha F_{3}$ is homogeneous of degree 3 one obtains, in view of (24),

$$
\tilde{H}_{V} \circ \Psi_{1}=G_{2}+\frac{1}{2}\left\{\alpha G_{3}, \alpha F_{3}\right\}+\beta G_{4}+O\left(\zeta^{5}\right) .
$$

Note that $\left\{G_{3}, F_{3}\right\}$ is homogeneous of order 4 . Hence our first step is achieved. It remains to solve (23). Since $G_{3}$ contains only monomials with $\left(k, k^{\prime}, k^{\prime \prime}\right) \in K_{3}$ (cf. (21)), also $F_{3}$ need only contain such monomials,

$$
F_{3}=\sum_{\left(k, k^{\prime}, k^{\prime \prime}\right) \in K_{3}} F_{k k^{\prime} k^{\prime \prime}}^{(3)} \zeta_{k} \zeta_{k^{\prime}} \zeta_{k^{\prime \prime}}
$$

which leads to

$$
\begin{aligned}
\left\{G_{2}, F_{3}\right\} & =i \sum_{1 \leq|k| \leq N-1} 2 \sigma_{k} \lambda_{k}^{2} \zeta_{-k} \frac{\partial F_{3}}{\partial \zeta_{-k}} \\
& =-i \sum_{\left(k, k^{\prime}, k^{\prime \prime}\right) \in K_{3}}\left(s_{k}+s_{k^{\prime}}+s_{k^{\prime \prime}}\right) F_{k k^{\prime} k^{\prime \prime}}^{(3)} \zeta_{k} \zeta_{k^{\prime}} \zeta_{k^{\prime \prime}}
\end{aligned}
$$


where

$$
s_{k}:=2 \sigma_{k} \lambda_{k}^{2}=2 \sin \frac{k \pi}{N}
$$

The following result is due to Beukers and Rink (cf. $[13,15])$ :

Lemma 4.1. For any $\left(k, k^{\prime}, k^{\prime \prime}\right) \in K_{3}$,

$$
s_{k}+s_{k^{\prime}}+s_{k^{\prime \prime}} \neq 0
$$

Let us remark that Lemma 4.1 also follows from the integrability of the Toda lattice (cf. [7]). We include the self-contained proof due to Beukers and Rink.

Proof. Suppose that $\left(k, k^{\prime}, k^{\prime \prime}\right) \in K_{3}$ satisfies $s_{k}+s_{k^{\prime}}+s_{k^{\prime \prime}}=0$. It follows from $k+$ $k^{\prime}+k^{\prime \prime} \equiv 0 \bmod N$ that either $s_{k^{\prime \prime}}=-s_{k+k^{\prime}}$ or $s_{k^{\prime \prime}}=s_{k+k^{\prime}}$, according to whether $k+k^{\prime}+k^{\prime \prime} \equiv 0$ or $k+k^{\prime}+k^{\prime \prime} \equiv N \bmod 2 N$.

In the first case, it follows that

$$
2 i \sin \frac{k \pi}{N}+2 i \sin \frac{k^{\prime} \pi}{N}-2 i \sin \left(\frac{k \pi}{N}+\frac{k^{\prime} \pi}{N}\right)=0 .
$$

Setting $x:=e^{\frac{i k \pi}{N}}$ and $y:=e^{\frac{i k^{\prime} \pi}{N}}$, one can rewrite (27) as

$$
0=x-\frac{1}{x}+y-\frac{1}{y}-x y+\frac{1}{x y}=(1-x)(1-y)(1-x y) \frac{1}{x y} .
$$

It follows that any solution of (28) contradicts the assumption $1 \leq|k|,\left|k^{\prime}\right|,\left|k^{\prime \prime}\right| \leq N-1$. Indeed, solutions with $x=1$ (i.e. $k \equiv 0 \bmod 2 N), y=1\left(\right.$ i.e. $k^{\prime} \equiv 0 \bmod 2 N$ ), or $x y=1$ (i.e. $k+k^{\prime} \equiv 0 \bmod 2 N$ and thus $k^{\prime \prime} \equiv 0 \bmod 2 N$ ), contradict this assumption.

In the second case, we have instead of (27),

$$
2 i \sin \frac{k \pi}{N}+2 i \sin \frac{k^{\prime} \pi}{N}+2 i \sin \left(\frac{k \pi}{N}+\frac{k^{\prime} \pi}{N}\right)=0 .
$$

With $x, y$ as above, it now follows from (29) that

$$
0=x-\frac{1}{x}+y-\frac{1}{y}+x y-\frac{1}{x y}=-(1+x)(1+y)(1-x y) \frac{1}{x y} .
$$

Again we conclude that any solution of (29) contradicts the assumption $1 \leq|k|,\left|k^{\prime}\right|$, $\left|k^{\prime \prime}\right| \leq N-1$. Indeed, solutions with $x=-1$ (i.e. $\left.k \equiv N \bmod 2 N\right), y=-1$ (i.e. $k^{\prime} \equiv N$ $\bmod 2 N)$, or $x y=1$ (i.e. $k+k^{\prime} \equiv 0 \bmod 2 N$ and thus $\left.k^{\prime \prime} \equiv N \bmod 2 N\right)$, contradict this assumption.

By Lemma 4.1, one can define $F_{3}$ as follows

$$
i F_{k k^{\prime} k^{\prime \prime}}^{(3)}:= \begin{cases}\frac{G_{k k^{\prime} k^{\prime \prime}}^{(3)}}{s_{k}+s_{k^{\prime}}+s_{k^{\prime \prime}}} & \left(k, k^{\prime}, k^{\prime \prime}\right) \in K_{3} \\ 0 & \text { otherwise. }\end{cases}
$$


Then $\left\{G_{2}, \alpha F_{3}\right\}+\alpha G_{3}=0$. Written more explicitly, the nonzero coefficients of $F_{3}$ are

$$
i F_{k k^{\prime} k^{\prime \prime}}^{(3)}=\frac{(-1)^{\left(k+k^{\prime}+k^{\prime \prime}\right) / N}}{6 \sqrt{N}} \frac{\sqrt{\left|\sin \frac{k \pi}{N} \sin \frac{k^{\prime} \pi}{N} \sin \frac{k^{\prime \prime} \pi}{N}\right|}}{2 \sin \frac{k \pi}{N}+2 \sin \frac{k^{\prime} \pi}{N}+2 \sin \frac{k^{\prime \prime} \pi}{N}} .
$$

In a second step we normalize the $4^{\text {th }}$ order term $\beta G_{4}+\frac{\alpha^{2}}{2}\left\{G_{3}, F_{3}\right\}$ in (25). We decompose this sum into its contribution to the Birkhoff normal form and the rest, to be transformed away in a moment. Let us first compute $\left\{G_{3}, F_{3}\right\}$ in a more explicit form:

$$
\begin{aligned}
& \left\{G_{3}, F_{3}\right\}=i \sum_{1 \leq|k| \leq N-1} \sigma_{k} \frac{\partial G_{3}}{\partial \zeta_{k}} \frac{\partial F_{3}}{\partial \zeta_{-k}}=\sum_{1 \leq|k| \leq N-1} \sigma_{k} \frac{\partial G_{3}}{\partial \zeta_{k}} \frac{\partial\left(i F_{3}\right)}{\partial \zeta_{-k}} \\
& =\frac{1}{N} \sum_{1 \leq|k| \leq N-1} \sigma_{k}\left(\frac{3}{6} \sum_{\substack{1 \leq|l|,|m| \leq N-1, l+m=-k+r N}}(-1)^{r} \lambda_{k} \lambda_{l} \lambda_{m} \zeta_{l} \zeta_{m}\right) \\
& \cdot\left(\frac{3}{6} \sum_{\substack{1 \leq\left|l^{\prime},\right| m^{\prime} \mid \leq N-1, l^{\prime}+m^{\prime}=k+r^{\prime} N}}(-1)^{r^{\prime}} \frac{\lambda_{k} \lambda_{l^{\prime}} \lambda_{m^{\prime}}}{s_{-k}+s_{l^{\prime}}+s_{m^{\prime}}} \zeta_{l^{\prime}} \zeta_{m^{\prime}}\right) \\
& =\frac{1}{8 N} \sum_{1 \leq|k| \leq N-1} \sum_{\substack{1 \leq|l|,|m|,\left|I^{\prime}\right|,\left|m^{\prime}\right| \leq N-1 \\
l+m-r N=-k \\
l^{\prime}+m^{\prime}-r^{\prime} N=k}}(-1)^{r+r^{\prime}} \frac{s_{k} \lambda_{l} \lambda_{m} \lambda_{l^{\prime}} \lambda_{m^{\prime}}}{s_{-k}+s_{l^{\prime}}+s_{m^{\prime}}} \zeta_{l} \zeta_{m} \zeta_{l^{\prime}} \zeta_{m^{\prime}},
\end{aligned}
$$

where for the latter equality we used that $2 \sigma_{k} \lambda_{k}^{2}=s_{k}$. Setting

$$
\varepsilon_{l m l^{\prime} m^{\prime}}:=\frac{l+m+l^{\prime}+m^{\prime}}{N}
$$

one then gets

$$
\begin{aligned}
& \left\{G_{3}, F_{3}\right\}=\frac{1}{8 N} \sum_{1 \leq|k| \leq N-1} \sum_{\substack{l+m \equiv=-k \bmod N \\
l^{\prime}+m^{\prime} \equiv k \bmod N}}(-1)^{\varepsilon_{l m l^{\prime} m^{\prime}}} \frac{\lambda_{l} \lambda_{m} \lambda_{l^{\prime}} \lambda_{m^{\prime}}}{-1+\left(s_{l^{\prime}}+s_{m^{\prime}}\right) / s_{k}} \zeta_{l} \zeta_{m} \zeta_{l^{\prime}} \zeta_{m^{\prime}} \\
& =\frac{1}{8 N} \sum_{k=1}^{N-1} \sum_{\substack{l+m \equiv-k \bmod N \\
l^{\prime}+m^{\prime} \equiv k \bmod N}}(-1)^{\varepsilon_{l m l^{\prime} m^{\prime}}} \frac{\lambda_{l} \lambda_{m} \lambda_{l^{\prime}} \lambda_{m^{\prime}}}{-1+\left(s_{l^{\prime}}+s_{m^{\prime}}\right) / s_{k}} \zeta_{l} \zeta_{m} \zeta_{l^{\prime}} \zeta_{m^{\prime}} \\
& +\frac{1}{8 N} \sum_{k=1}^{N-1} \sum_{\substack{l+m \equiv k \bmod N \\
l^{\prime}+m^{\prime} \equiv-k \bmod N}}(-1)^{\varepsilon_{l m l^{\prime} m^{\prime}}} \frac{\lambda_{l} \lambda_{m} \lambda_{l^{\prime}} \lambda_{m^{\prime}}}{-1-\left(s_{l^{\prime}}+s_{m^{\prime}}\right) / s_{k}} \zeta_{l} \zeta_{m} \zeta_{l^{\prime}} \zeta_{m^{\prime}} \\
& =\frac{1}{8 N} \sum_{k=1}^{N-1} \sum_{\substack{l+m \equiv-k \bmod N \\
l^{\prime}+m^{\prime} \equiv k \bmod N}}\left(\frac{1}{-1+\left(s_{l^{\prime}}+s_{m^{\prime}}\right) / s_{k}}+\frac{1}{-1-\left(s_{l}+s_{m}\right) / s_{k}}\right) \\
& \cdot(-1)^{\varepsilon_{l m l^{\prime} m^{\prime}}} \lambda_{l} \lambda_{m} \lambda_{l^{\prime}} \lambda_{m^{\prime}} \zeta_{l} \zeta_{m} \zeta_{l^{\prime}} \zeta_{m^{\prime}}
\end{aligned}
$$

Note that for $k=l^{\prime}+m^{\prime}+r^{\prime} N$ with $1 \leq k \leq N-1$ and $r^{\prime} \in \mathbb{Z}$ we have

$$
s_{k}=\left|s_{l^{\prime}+m^{\prime}}\right| \text {. }
$$


Introduce $^{1}$ for any $\left(l, m, l^{\prime}, m^{\prime}\right) \in K_{4}$,

$$
c_{l m l^{\prime} m^{\prime}}= \begin{cases}\frac{1}{-1+\frac{s_{l^{\prime}+s^{\prime}}}{s_{l^{\prime}+m^{\prime}} \mid}}-\frac{1}{1+\frac{s l^{+}+s_{m}}{\left|s_{l+m}\right|}} & \text { if } l+m \neq \equiv \bmod N \\ 0 & \text { otherwise. }\end{cases}
$$

We then get

$$
\frac{\alpha^{2}}{2}\left\{G_{3}, F_{3}\right\}=\frac{\alpha^{2}}{16 N} \sum_{\left(l, m, l^{\prime}, m^{\prime}\right) \in K_{4}} c_{l m l^{\prime} m^{\prime}}(-1)^{\varepsilon_{l m l^{\prime} m^{\prime}}} \lambda_{l} \lambda_{m} \lambda_{l^{\prime}} \lambda_{m^{\prime}} \zeta_{l} \zeta_{m} \zeta_{l^{\prime}} \zeta_{m^{\prime}}
$$

Combined with formula (20) for $G_{4}$, the quantity $\beta G_{4}+\frac{\alpha^{2}}{2}\left\{G_{3}, F_{3}\right\}$ becomes

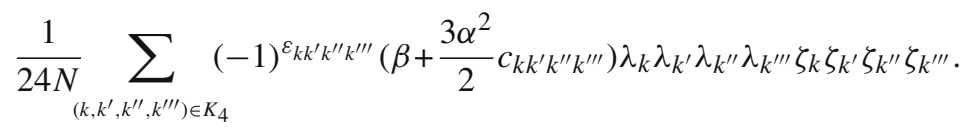

We now decompose (33) into its contribution to the Birkhoff normal form of $H_{V}$ and the rest, and we denote by $\pi_{N}$ the projection onto the former one, whereas the latter one will be (partially) transformed away by a second transformation $\Psi_{2}$.

Lemma 4.2. The normal form part of $\beta G_{4}+\frac{\alpha^{2}}{2}\left\{G_{3}, F_{3}\right\}$ is given by

$$
\begin{aligned}
& \pi_{N}\left(\beta G_{4}+\frac{\alpha^{2}}{2}\left\{G_{3}, F_{3}\right\}\right) \\
& =\frac{1}{4 N}\left(\sum_{l=1}^{N-1}\left(\alpha^{2}+\left(\beta-\alpha^{2}\right) \lambda_{l}^{4}\right)\left|\zeta_{l}\right|^{4}+2 \sum_{1 \leq l \neq m \leq N-1}\left(\beta-\alpha^{2}\right) \lambda_{l}^{2} \lambda_{m}^{2}\left|\zeta_{l}\right|^{2}\left|\zeta_{m}\right|^{2}\right) .
\end{aligned}
$$

Proof. The indices $k, k^{\prime}, k^{\prime \prime}, k^{\prime \prime \prime}$ of the terms in $\beta G_{4}+\frac{\alpha^{2}}{2}\left\{G_{3}, F_{3}\right\}$ contributing to the normal form satisfy $\left(k, k^{\prime}, k^{\prime \prime}, k^{\prime \prime \prime}\right) \in K_{4}^{N}$, where

$$
\begin{array}{r}
K_{4}^{N}:=\left\{\left(k, k^{\prime}, k^{\prime \prime}, k^{\prime \prime \prime}\right) \in K_{4} \mid \exists 1 \leq l \leq m \leq N-1\right. \text { such that } \\
\left.\left\{k, k^{\prime}, k^{\prime \prime}, k^{\prime \prime \prime}\right\}=\{l,-l, m,-m\}\right\} .
\end{array}
$$

In the case $l=m,\{l,-l, l,-l\}$ in (35) is viewed as a set-like object whose two elements $l$ and $-l$ each have multiplicity two.

We investigate $\pi_{N}\left(\beta G_{4}\right)$ and $\pi_{N}\left(\frac{\alpha^{2}}{2}\left\{G_{3}, F_{3}\right\}\right)$ separately. Let us start with $\beta G_{4}$. We distinguish the cases $l=m$ and $l \neq m$ in $K_{4}^{N}$. For $l=m$, there are $\left(\begin{array}{l}4 \\ 2\end{array}\right)=6$ distinct permutations of $\left(k, k^{\prime}, k^{\prime \prime}, k^{\prime \prime \prime}\right)$ in $K_{4}^{N}$, whereas for $l \neq m$, all $4 !=24$ permutations of $(l, m,-l,-m)$ are distinct. Hence we have

$$
\begin{aligned}
\pi_{N}\left(\beta G_{4}\right) & =\frac{\beta}{24 N}\left(6 \sum_{l=1}^{N-1} \lambda_{l}^{4}\left|\zeta_{l}\right|^{4}+24 \sum_{1 \leq l<m \leq N-1} \lambda_{l}^{2} \lambda_{m}^{2}\left|\zeta_{l}\right|^{2}\left|\zeta_{m}\right|^{2}\right) \\
& =\frac{\beta}{4 N}\left(\sum_{l=1}^{N-1} \lambda_{l}^{4}\left|\zeta_{l}\right|^{4}+2 \sum_{1 \leq l \neq m \leq N-1} \lambda_{l}^{2} \lambda_{m}^{2}\left|\zeta_{l}\right|^{2}\left|\zeta_{m}\right|^{2}\right) .
\end{aligned}
$$

\footnotetext{
${ }^{1}$ To keep the formula for $c_{l m l^{\prime} m^{\prime}}$ as simple as possible we have not symmetrized the coefficients $c_{l m l^{\prime} m^{\prime}}$.
} 
Now let us compute $\pi_{N}\left(\frac{\alpha^{2}}{2}\left\{G_{3}, F_{3}\right\}\right)$. We have to single out the matches of (35) for which in addition the coefficient $c_{k k^{\prime} k^{\prime \prime} k^{\prime \prime \prime}}$ in (32) does not vanish, i.e.

$$
k+k^{\prime} \not \equiv 0 \bmod N \text { and } k+k^{\prime}+k^{\prime \prime}+k^{\prime \prime \prime} \equiv 0 \bmod N
$$

There are two quadruples $\left(k, k^{\prime}, k^{\prime \prime}, k^{\prime \prime \prime}\right)$ in $K_{4}^{N}$ which satisfy these additional conditions,

$$
\begin{aligned}
k+k^{\prime \prime} & =0 \\
k^{\prime}+k^{\prime \prime \prime} & =0
\end{aligned} \quad \text { or } \quad \begin{aligned}
& k+k^{\prime \prime \prime}=0 \\
& k^{\prime}+k^{\prime \prime}=0
\end{aligned} .
$$

In both cases, we have $s_{k^{\prime \prime}}+s_{k^{\prime \prime \prime}}=-\left(s_{k}+s_{k^{\prime}}\right)$, and therefore (31) reduces to

$$
c_{k k^{\prime} k^{\prime \prime} k^{\prime \prime \prime}}=\frac{-2\left|s_{k+k^{\prime}}\right|}{\left|s_{k+k^{\prime}}\right|+s_{k}+s_{k^{\prime}}} \text {. }
$$

Note that (38) remains valid for $k+k^{\prime}=N$, since in this case $s_{k+k^{\prime}}=0$ and $s_{k}+s_{k^{\prime}}>0$ as $k$ and $k^{\prime}$ must satisfy $1 \leq k, k^{\prime} \leq N-1$, but not for $k+k^{\prime}=0$, since in this case $\left|s_{k+k^{\prime}}\right|+s_{k}+s_{k^{\prime}}=0$.

We first compute the diagonal part of $\pi_{N}\left(\frac{1}{2}\left\{G_{3}, F_{3}\right\}\right)$. In this case, the two possibilities in (37) coincide and the solutions are

$$
\left(k, k^{\prime}, k^{\prime \prime}, k^{\prime \prime \prime}\right)=\left\{\begin{array}{c}
(l, \quad l,-l,-l) \\
(-l,-l, l, \quad l)
\end{array}\right.
$$

where $1 \leq l \leq N-1$. The sum of the coefficients $c_{k k^{\prime} k^{\prime \prime} k^{\prime \prime \prime}}$ for the two cases listed in (39) is

$$
c_{l, l,-l,-l}+c_{-l,-l, l, l}=-2\left|s_{2 l}\right|\left(\frac{1}{\left|s_{2 l}\right|+2 s_{l}}+\frac{1}{\left|s_{2 l}\right|-2 s_{l}}\right)=\frac{-4 s_{2 l}^{2}}{s_{2 l}^{2}-4 s_{l}^{2}}=4 \cot ^{2} \frac{l \pi}{N} .
$$

We now turn to the off-diagonal part of $\pi_{N}\left(\frac{1}{2}\left\{G_{3}, F_{3}\right\}\right)$. The quadruples $\left(k, k^{\prime}\right.$, $\left.k^{\prime \prime}, k^{\prime \prime \prime}\right) \in K_{4}$ satisfying (37) for given $\{l, m\} \subseteq\{1, \ldots, N-1\}$ with $l<m,\left(k, k^{\prime}\right)=$ $( \pm l, \pm m)$, and $\left(k^{\prime \prime}, k^{\prime \prime \prime}\right)=( \pm l, \pm m)$, are

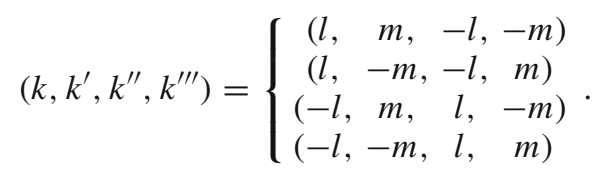

The remaining matches are obtained from (40) by permuting the first and second or the third and fourth columns on the right-hand side of (40), bringing the total number of all matches to $16=4 \cdot 4$. Note that by formula (38), these permutations leave the value of

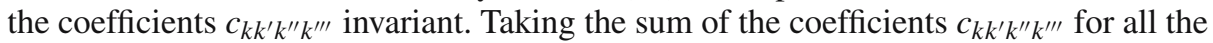


quadruples listed in (40), we obtain

$$
\begin{aligned}
& 4\left(c_{l, m,-l,-m}+c_{l,-m,-l, m}+c_{-l, m, l,-m}+c_{-l,-m, l, m}\right) \\
&=-8\left(\frac{\left|s_{l+m}\right|}{\left|s_{l+m}\right|+s_{l}+s_{m}}+\frac{\left|s_{l-m}\right|}{\left|s_{l-m}\right|+s_{l}-s_{m}}\right. \\
&\left.+\frac{\left|s_{l-m}\right|}{\left|s_{l-m}\right|-s_{l}+s_{m}}+\frac{\left|s_{l+m}\right|}{\left|s_{l+m}\right|-s_{l}-s_{m}}\right) \\
&=-16\left(\frac{s_{l-m}^{2}}{s_{l-m}^{2}-\left(s_{l}-s_{m}\right)^{2}}+\frac{s_{l+m}^{2}}{s_{l+m}^{2}-\left(s_{l}+s_{m}\right)^{2}}\right) \\
&= \frac{-16\left(2 s_{l-m}^{2} s_{l+m}^{2}-s_{l-m}^{2}\left(s_{l}+s_{m}\right)^{2}-s_{l+m}^{2}\left(s_{l}-s_{m}\right)^{2}\right)}{s_{l-m}^{2} s_{l+m}^{2}+\left(s_{l}-s_{m}\right)^{2}\left(s_{l}+s_{m}\right)^{2}-s_{l-m}^{2}\left(s_{l}+s_{m}\right)^{2}-s_{l+m}^{2}\left(s_{l}-s_{m}\right)^{2}}, \\
&=-16,
\end{aligned}
$$

since $s_{l-m}^{2} s_{l+m}^{2}=\left(s_{l}-s_{m}\right)^{2}\left(s_{l}+s_{m}\right)^{2}$. Collecting terms, we thus have

$$
\begin{aligned}
\pi_{N}\left(\frac{\alpha^{2}}{2}\left\{G_{3}, F_{3}\right\}\right) & =\frac{\alpha^{2}}{16 N}\left(\sum_{l=1}^{N-1} 4 \cos ^{2} \frac{\pi l}{N}\left|\zeta_{l}\right|^{4}-16 \sum_{1 \leq l<m \leq N-1} \lambda_{l}^{2} \lambda_{m}^{2}\left|\zeta_{l}\right|^{2}\left|\zeta_{m}\right|^{2}\right) \\
& =\frac{\alpha^{2}}{4 N}\left(\sum_{l=1}^{N-1}\left(1-\lambda_{l}^{4}\right)\left|\zeta_{l}\right|^{4}-2 \sum_{1 \leq l \neq m \leq N-1} \lambda_{l}^{2} \lambda_{m}^{2}\left|\zeta_{l}\right|^{2}\left|\zeta_{m}\right|^{2}\right)
\end{aligned}
$$

Adding up (36) and (41), we obtain (34).

Now we want to remove [as much as possible of] the term (Id $\left.-\pi_{N}\right)\left(\beta G_{4}+\right.$ $\left.\frac{\alpha^{2}}{2}\left\{G_{3}, F_{3}\right\}\right)$ from the Hamiltonian $(25), \tilde{H}_{V} \circ \Psi_{1}$, by a second coordinate transformation $\Psi_{2}$. In view of formulas (20) and (32) for $G_{4}$ and $\frac{1}{2}\left\{G_{3}, F_{3}\right\}$, respectively, and in complete analogy to the first step we look for a transformation $\Psi_{2}$ of the form $\Psi_{2}=\left.X_{F_{4}}^{t}\right|_{t=1}$ with

$$
F_{4}=\sum_{\left(k, k^{\prime}, k^{\prime \prime}, k^{\prime \prime \prime}\right) \in K_{4} \backslash K_{4}^{N}} F_{k k^{\prime} k^{\prime \prime} k^{\prime \prime \prime}}^{(4)} \zeta_{k} \zeta_{k^{\prime}} \zeta_{k^{\prime \prime}} \zeta_{k^{\prime \prime \prime}}
$$

where $F_{\sigma\left(k, k^{\prime}, k^{\prime \prime}, k^{\prime \prime \prime}\right)}^{(4)}=F_{\left(k, k^{\prime}, k^{\prime \prime}, k^{\prime \prime \prime}\right)}^{(4)}$ for any permutation $\sigma\left(k, k^{\prime}, k^{\prime \prime}, k^{\prime \prime \prime}\right)$ of the quadruple $\left(k, k^{\prime}, k^{\prime \prime}, k^{\prime \prime \prime}\right) \in K_{4} \backslash K_{4}^{N}$. We would like to determine the coefficients of $F_{4}$ in such a way that

$$
\left\{G_{2}, F_{4}\right\}=-\left(\mathrm{Id}-\pi_{N}\right)\left(\beta G_{4}+\frac{\alpha^{2}}{2}\left\{G_{3}, F_{3}\right\}\right)
$$

As in (26) one gets

$$
\left\{G_{2}, F_{4}\right\}=-i \sum_{\left(k, k^{\prime}, k^{\prime \prime}, k^{\prime \prime \prime}\right) \in K_{4} \backslash K_{4}^{N}}\left(s_{k}+s_{k^{\prime}}+s_{k^{\prime \prime}}+s_{k^{\prime \prime \prime}}\right) F_{k k^{\prime} k^{\prime \prime} k^{\prime \prime \prime}}^{(4)} \zeta_{k} \zeta_{k^{\prime}} \zeta_{k^{\prime \prime}} \zeta_{k^{\prime \prime \prime}}
$$


and Eq. (42) combined with (33) leads to

$$
\begin{aligned}
& i\left(s_{k}+s_{k^{\prime}}+s_{k^{\prime \prime}}+s_{k^{\prime \prime \prime}}\right) F_{k k^{\prime} k^{\prime \prime} k^{\prime \prime \prime}}^{(4)} \\
& \quad=\frac{1}{24 N}(-1)^{\varepsilon_{k k^{\prime} k^{\prime \prime} k^{\prime \prime \prime}}}\left(\beta+\frac{3 \alpha^{2}}{2} c_{k k^{\prime} k^{\prime \prime} k^{\prime \prime \prime}}^{S}\right) \cdot \lambda_{k} \lambda_{k^{\prime}} \lambda_{k^{\prime \prime}} \lambda_{k^{\prime \prime \prime}}
\end{aligned}
$$

for any quadruple $\left(k, k^{\prime}, k^{\prime \prime}, k^{\prime \prime \prime}\right)$ in $K_{4} \backslash K_{4}^{N}$. Here $c_{k k^{\prime} k^{\prime \prime} k^{\prime \prime \prime}}^{S}$ denotes the symmetrized

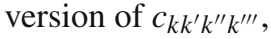

$$
c_{k k^{\prime} k^{\prime \prime} k^{\prime \prime \prime}}^{S}:=\frac{1}{4 !} \sum_{\sigma \in S_{4}} c_{\sigma\left(k, k^{\prime}, k^{\prime \prime}, k^{\prime \prime \prime}\right)} .
$$

The following lemma due to Beukers and Rink (cf. [13]) determines the quadruples $\left(k, k^{\prime}, k^{\prime \prime}, k^{\prime \prime \prime}\right) \in K_{4} \backslash K_{4}^{N}$ for which $s_{k}+s_{k^{\prime}}+s_{k^{\prime \prime}}+s_{k^{\prime \prime \prime}}=0$. Let us introduce

$$
K_{4}^{r e s}:=K_{r e s}^{+} \cup K_{r e s}^{-} \subseteq K_{4},
$$

where

$$
\begin{aligned}
K_{\text {res }}^{ \pm}:=\left\{\left(k, k^{\prime}, k^{\prime \prime}, k^{\prime \prime \prime}\right) \in K_{4} \mid \exists l \in \mathbb{N}: 1 \leq l \leq \frac{N}{4}\right. \text { so that } \\
\left.\left\{k, k^{\prime}, k^{\prime \prime}, k^{\prime \prime \prime}\right\}=\left\{ \pm l, \pm l \mp N, \frac{N}{2} \mp l,-\frac{N}{2} \mp l\right\}\right\} .
\end{aligned}
$$

Note that if $N$ is odd, then $K_{4}^{r e s}=\emptyset$.

Lemma 4.3. Let $\left(k_{1}, k_{2}, k_{3}, k_{4}\right) \in K_{4} \backslash K_{4}^{N}$. Then

$$
s_{k}+s_{k^{\prime}}+s_{k^{\prime \prime}}+s_{k^{\prime \prime \prime}}=0 \text { if and only if }\left(k, k^{\prime}, k^{\prime \prime}, k^{\prime \prime \prime}\right) \in K_{4}^{r e s} .
$$

In particular, if $N$ is odd, then $s_{k}+s_{k^{\prime}}+s_{k^{\prime \prime}}+s_{k^{\prime \prime \prime}} \neq 0$.

For the convenience of the reader a detailed proof of Lemma 4.3 is given in Appendix A. It is likely that Lemma 4.3 also can be proved using the integrability of the Toda lattice (cf. [7]).

By Lemma 4.3, if $N$ is odd, (44) can be solved for any $\left(k, k^{\prime}, k^{\prime \prime}, k^{\prime \prime \prime}\right) \in K_{4} \backslash K_{4}^{N}$ determining the coefficients $F_{k k^{\prime} k^{\prime \prime} k^{\prime \prime \prime}}^{(4)}$ with $\left(k, k^{\prime}, k^{\prime \prime}, k^{\prime \prime \prime}\right) \in K_{4} \backslash K_{4}^{N}$ in such a way that $F_{\sigma\left(k, k^{\prime}, k^{\prime \prime}, k^{\prime \prime \prime}\right)}^{(4)}=F_{\left(k, k^{\prime}, k^{\prime \prime}, k^{\prime \prime \prime}\right)}^{(4)}$ for any permutation $\sigma\left(k, k^{\prime}, k^{\prime \prime}, k^{\prime \prime \prime}\right)$ of $\left(k, k^{\prime}, k^{\prime \prime}, k^{\prime \prime \prime}\right) \in$ $K_{4} \backslash K_{4}^{N}$. With this choice of $F_{4}$ the canonical transformation $\Psi_{2}$ is then defined by $\left.X_{F_{4}}^{t}\right|_{t=1}$. Composing $\Psi_{1}$ and $\Psi_{2}$, we obtain the transformation $\Xi:=\Psi_{1} \circ \Psi_{2}$. We have proved the following

Proposition 4.4. Assume that $N \geq 3$ is odd. The real analytic symplectic coordinate transformation $\zeta=\Xi(z)$, defined in a neighborhood of the origin in $Z$, transforms the Hamiltonian $\tilde{H}_{V}$ into its Birkhoff normal form up to order 4. More precisely,

$$
\tilde{H}_{V} \circ \Xi=G_{2}+\pi_{N}\left(\beta G_{4}+\frac{\alpha^{2}}{2}\left\{G_{3}, F_{3}\right\}\right)+O\left(z^{5}\right),
$$

with $G_{2}$ and $\pi_{N}\left(\beta G_{4}+\frac{\alpha^{2}}{2}\left\{G_{3}, F_{3}\right\}\right)$ given by (18) and (34), respectively. 
Theorem 1.1 can now be proved easily.

Proof of Theorem 1.1. Proposition 4.4 provides the Taylor series expansion of $\tilde{H}_{V}$ in terms of the actions

$$
I=\left(I_{k}\right)_{1 \leq k \leq N-1}, \quad I_{k}=\frac{x_{k}^{2}+y_{k}^{2}}{2} .
$$

More precisely, $\tilde{H}_{V} \circ \Xi=H_{\alpha, \beta}(I)+O\left(z^{5}\right)$, where $H_{\alpha, \beta}(I)$ is defined by

$$
2 \sum_{k=1}^{N-1} \lambda_{k}^{2} I_{k}+\frac{1}{4 N} \sum_{k=1}^{N-1}\left(\alpha^{2}+\left(\beta-\alpha^{2}\right) \lambda_{k}^{4}\right) I_{k}^{2}+\frac{\beta-\alpha^{2}}{2 N} \sum_{\substack{l \neq m \\ 1 \leq l, m \leq N-1}} \lambda_{l}^{2} \lambda_{m}^{2} I_{l} I_{m}
$$

and $\lambda_{k}=\left|\sin \frac{k \pi}{N}\right|^{\frac{1}{2}}$. This proves Theorem 1.1.

\section{Proof of Theorem 1.4.}

Now we assume that $N$ is even. To obtain the normal form of the FPU Hamiltonian as claimed in Theorem 1.4 we continue the investigations of the previous section. According to Lemma 4.3, Eq. (44) might have no solution $F_{k k^{\prime} k^{\prime \prime} k^{\prime \prime \prime}}^{(4)}$ for $\left(k, k^{\prime}, k^{\prime \prime}, k^{\prime \prime \prime}\right) \in K_{4}^{r e s}$. We first compute the projection $\pi_{r e s}\left(\beta G_{4}+\frac{\alpha^{2}}{2}\left\{G_{3}, F_{3}\right\}\right)$ of $\beta G_{4}+\frac{\alpha^{2}}{2}\left\{G_{3}, F_{3}\right\}$ onto those terms which are indexed by quadruples $\left(k, k^{\prime}, k^{\prime \prime}, k^{\prime \prime \prime}\right) \in K_{4}^{r e s}$, i.e. the projection onto the resonant non-normal form part of $\beta G_{4}+\frac{\alpha^{2}}{2}\left\{G_{3}, F_{3}\right\}$.

Lemma 5.1. Assume that $N$ is even. The resonant non-normal form part of $\beta G_{4}+$ $\frac{\alpha^{2}}{2}\left\{G_{3}, F_{3}\right\}$ is given by

$$
\pi_{r e s}\left(\beta G_{4}+\frac{\alpha^{2}}{2}\left\{G_{3}, F_{3}\right\}\right)=-\frac{\beta-\alpha^{2}}{4 N}\left(R+R_{\frac{N}{4}}\right),
$$

where

$$
R=\sum_{1 \leq l<\frac{N}{4}} s_{2 l}\left(\zeta_{l} \zeta_{-N+l} \zeta_{\frac{N}{2}-l} \zeta_{-\frac{N}{2}-l}+\zeta_{-l} \zeta_{N-l} \zeta_{\frac{N}{2}+l} \zeta_{-\frac{N}{2}+l}\right)
$$

and

$$
R_{\frac{N}{4}}= \begin{cases}\frac{1}{2}\left(\zeta_{\frac{N}{4}}^{2} \zeta_{-\frac{3 N}{4}}^{2}+\zeta_{\frac{3 N}{4}}^{2} \zeta_{-\frac{N}{4}}^{2}\right) & \text { if } \frac{N}{4} \in \mathbb{N} \\ 0 & \text { otherwise }\end{cases}
$$

Proof. Consider the formula (33) for $\beta G_{4}+\frac{\alpha^{2}}{2}\left\{G_{3}, F_{3}\right\}$. At this point we need to consider the symmetrized version (45) of the coefficients $c_{k l k^{\prime} l^{\prime}}$ defined by (31). We claim that for any $\left(k_{1}, k_{2}, k_{3}, k_{4}\right) \in K_{4}^{r e s}$,

$$
c_{k_{1} k_{2} k_{3} k_{4}}^{S}=\frac{1}{4 !} \sum_{\sigma \in S_{4}} c_{k_{\sigma(1)} k_{\sigma(2)} k_{\sigma(3)} k_{\sigma(4)}}=-\frac{2}{3} .
$$


Observe that $c_{k_{1} k_{2} k_{3} k_{4}}$ is invariant under the transpositions $k_{1} \leftrightarrow k_{2}$ and $k_{3} \leftrightarrow k_{4}$. Hence (52) follows once we prove that

$$
4\left(c_{k_{1} k_{2} k_{3} k_{4}}+c_{k_{1} k_{3} k_{2} k_{4}}+c_{k_{1} k_{4} k_{2} k_{3}}+c_{k_{2} k_{4} k_{1} k_{3}}+c_{k_{2} k_{3} k_{1} k_{4}}+c_{k_{3} k_{4} k_{1} k_{2}}\right)=-16 .
$$

Note that any element $\left(k_{1}, k_{2}, k_{3}, k_{4}\right) \in K_{4}^{r e s}$ is, $\bmod 2 N$, a permutation of an element of the form $(l,-N+l, N / 2-l,-N / 2-l)$ with $1 \leq|l| \leq N / 4$. For such quadruples one gets by a straightforward computation

$$
c_{k_{1} k_{2} k_{3} k_{4}}+c_{k_{3} k_{4} k_{1} k_{2}}=-2-2=-4
$$

and, with $c_{l}=2 \cos \frac{l \pi}{N}$,

$$
c_{k_{1} k_{3} k_{2} k_{4}}+c_{k_{2} k_{4} k_{1} k_{3}}=-\frac{4}{2+\left(s_{l}+c_{l}\right)}-\frac{4}{2-\left(s_{l}+c_{l}\right)}=-\frac{8}{s_{2 l}}
$$

as well as

$$
c_{k_{1} k_{4} k_{2} k_{3}}+c_{k_{2} k_{3} k_{1} k_{4}}=-\frac{4}{2+\left(s_{l}-c_{l}\right)}-\frac{4}{2-\left(s_{l}-c_{l}\right)}=\frac{8}{s_{2 l}} .
$$

Substituting these three identities into the left hand side of (53) leads to the claimed identity (53).

Moreover, by the definition (30) of $\varepsilon_{l m l^{\prime} m^{\prime}}$ one has for any $\left(k_{1}, k_{2}, k_{3}, k_{4}\right) \in K_{4}^{r e s}$ and any $\sigma \in S_{4}$, that $\varepsilon_{\sigma\left(k_{1}, k_{2}, k_{3}, k_{4}\right)}= \pm 1$ and hence

$$
(-1)^{\varepsilon_{\sigma\left(k_{1}, k_{2}, k_{3}, k_{4}\right)}}=-1 \text {. }
$$

Further,

$$
\lambda_{k_{1}} \lambda_{k_{2}} \lambda_{k_{3}} \lambda_{k_{4}}=\left|\sin \frac{l \pi}{N} \cos \frac{l \pi}{N}\right|=\frac{1}{2}\left|\sin \frac{2 l \pi}{N}\right|=\frac{1}{4}\left|s_{2 l}\right| .
$$

Combining all these computations we get

$$
\begin{aligned}
& \pi_{r e s}\left(\beta G_{4}+\frac{\alpha^{2}}{2}\left\{G_{3}, F_{3}\right\}\right) \\
& =\frac{1}{24 N} \sum_{\left(k_{1}, k_{2}, k_{3}, k_{4}\right) \in K_{4}^{\text {res }}}(-1)\left(\beta+\frac{3 \alpha^{2}}{2} c_{k_{1} k_{2} k_{3} k_{4}}^{S}\right) \lambda_{k_{1}} \lambda_{k_{2}} \lambda_{k_{3}} \lambda_{k_{4}} \zeta_{k_{1}} \zeta_{k_{2}} \zeta_{k_{3}} \zeta_{k_{4}} \\
& =-\frac{4 !\left(\beta-\alpha^{2}\right)}{24 N} \sum_{1 \leq l<\frac{N}{4}} \frac{s_{2 l}}{4}\left(\zeta_{l} \zeta_{-N+l} \zeta_{\frac{N}{2}-l} \zeta_{-\frac{N}{2}-l}+\zeta_{-l} \zeta_{N-l} \zeta_{\frac{N}{2}+l} \zeta_{-\frac{N}{2}+l}\right) \\
& \underbrace{-\frac{3 !\left(\beta-\alpha^{2}\right)}{24 N} \cdot \frac{2}{4}\left(\zeta_{\frac{N}{4}}^{2} \zeta_{-\frac{3 N}{4}}^{2}+\zeta_{-\frac{N}{4}}^{2} \zeta_{\frac{3 N}{4}}^{2}\right)}_{\text {only if } \frac{N}{4} \in \mathbb{N}} \\
& =-\frac{\beta-\alpha^{2}}{4 N}\left(R+R_{\frac{N}{4}}\right)
\end{aligned}
$$

with $R$ and $R_{\frac{N}{4}}$ as defined by (50) and (51), respectively. Hence Lemma 5.1 is proved. 
By Lemma 5.1, if $N$ is even, Eq. (44) can be solved for any quadruple $\left(k, k^{\prime}, k^{\prime \prime}, k^{\prime \prime \prime}\right) \in$ $K_{4} \backslash\left(K_{4}^{N} \cup K_{4}^{r e s}\right)$ in such a way that $F_{\sigma\left(k, k^{\prime}, k^{\prime \prime}, k^{\prime \prime \prime}\right)}^{(4)}=F_{\left(k, k^{\prime}, k^{\prime \prime}, k^{\prime \prime \prime}\right)}^{(4)}$ for any permutation $\sigma\left(k, k^{\prime}, k^{\prime \prime}, k^{\prime \prime \prime}\right)$ of $\left(k, k^{\prime}, k^{\prime \prime}, k^{\prime \prime \prime}\right)$. With this choice of $F_{4}$ the canonical transformation $\Psi_{2}$ is then defined by $\left.X_{F_{4}}^{t}\right|_{t=1}$. Composing $\Psi_{1}$ and $\Psi_{2}$, we obtain the transformation $\Xi:=\Psi_{1} \circ \Psi_{2}$ and have proved the following

Proposition 5.2. Assume that $N$ is even. The real analytic symplectic coordinate transformation $\zeta=\Xi(z)$, defined locally in a neighborhood of the origin $z=0$ in $z$, transforms the Hamiltonian $\tilde{H}_{V}$ into the resonant Birkhoff normal form up to order 4 ,

$$
\tilde{H}_{V} \circ \Xi=G_{2}+\pi_{N}\left(\beta G_{4}+\frac{\alpha^{2}}{2}\left\{G_{3}, F_{3}\right\}\right)+\pi_{r e s}\left(\beta G_{4}+\frac{\alpha^{2}}{2}\left\{G_{3}, F_{3}\right\}\right)+O\left(z^{5}\right),
$$

with $G_{2}, \pi_{N}\left(\beta G_{4}+\frac{\alpha^{2}}{2}\left\{G_{3}, F_{3}\right\}\right)$, and $\pi_{r e s}\left(\beta G_{4}+\frac{\alpha^{2}}{2}\left\{G_{3}, F_{3}\right\}\right)$ given by (18), (34), and (49), respectively.

Proof of Theorem 1.4. We start with the formula for $\tilde{H}_{V} \circ \Xi$ given by Proposition 5.2 and treat the normal form terms $G_{2}+\pi_{N}\left(\beta G_{4}+\frac{\alpha^{2}}{2}\left\{G_{3}, F_{3}\right\}\right)$ and the resonant normal form terms $\pi_{r e s}\left(\beta G_{4}+\frac{\alpha^{2}}{2}\left\{G_{3}, F_{3}\right\}\right)$ separately. With the action variables $I=\left(I_{k}\right)_{1 \leq k \leq N-1}$ defined by (47) we see that $G_{2}+\pi_{N}\left(\beta G_{4}+\frac{\alpha^{2}}{2}\left\{G_{3}, F_{3}\right\}\right)=H_{\alpha, \beta}(I)$, where $H_{\alpha, \beta}(I)$ is defined by (48). Concerning $\pi_{r e s}\left(\beta G_{4}+\frac{\alpha^{2}}{2}\left\{G_{3}, F_{3}\right\}\right)$, we first express it in terms of the real variables $\left(x_{k}, y_{k}\right)_{1 \leq k \leq N-1}$, related to the $\zeta_{k}$ 's by $x_{k}=\left(\zeta_{k}+\zeta_{-k}\right) / 2$ and $y_{k}=\left(\zeta_{-k}-\zeta_{k}\right) / 2 i$. Note that

$$
\begin{aligned}
& \zeta_{l} \zeta_{-N+l} \zeta_{\frac{N}{2}-l} \zeta_{-\frac{N}{2}-l}+\zeta_{-l} \zeta_{N-l} \zeta_{-\frac{N}{2}+l} \zeta_{\frac{N}{2}+l} \\
&= 2 \operatorname{Re}\left(\zeta_{l} \zeta_{-N+l} \zeta_{\frac{N}{2}-l} \zeta_{-\frac{N}{2}-l}\right) \\
&= \frac{1}{2}\left(\left(x_{l} x_{N-l}+y_{l} y_{N-l}\right)\left(x_{\frac{N}{2}-l} x_{\frac{N}{2}+l}+y_{\frac{N}{2}-l} y_{\frac{N}{2}+l}\right)\right. \\
&\left.-\left(x_{l} y_{N-l}-x_{N-l} y_{l}\right)\left(x_{\frac{N}{2}-l} y_{\frac{N}{2}+l}-x_{\frac{N}{2}+l} y_{\frac{N}{2}-l}\right)\right) \\
&= 2\left(J_{l} J_{\frac{N}{2}-l}-M_{l} M_{\frac{N}{2}-l}\right)
\end{aligned}
$$

where for any $1 \leq k \leq N-1$,

$$
J_{k}=\frac{1}{2}\left(x_{k} x_{N-k}+y_{k} y_{N-k}\right) \text { and } M_{k}=\frac{1}{2}\left(x_{k} y_{N-k}-x_{N-k} y_{k}\right) .
$$

Hence $R$, given by (50), can be expressed in terms of $J_{k}$ and $M_{k}$ as follows:

$$
\begin{aligned}
R(J, M) & =\sum_{1 \leq l<\frac{N}{4}} s_{2 l}\left(\zeta_{l} \zeta_{-N+l} \zeta_{\frac{N}{2}-l} \zeta_{-\frac{N}{2}-l}+\zeta_{-l} \zeta_{N-l} \zeta_{\frac{N}{2}+l} \zeta_{-\frac{N}{2}+l}\right) \\
& =4 \sum_{1 \leq l<\frac{N}{4}} \sin \frac{2 l \pi}{N}\left(J_{l} J_{\frac{N}{2}-l}-M_{l} M_{\frac{N}{2}-l}\right) .
\end{aligned}
$$

Similarly, if $\frac{N}{4} \in \mathbb{N}$, one concludes from (55) that $R_{\frac{N}{4}}$, given by (51), can be expressed as

$$
R_{\frac{N}{4}}(J, M)=\frac{1}{2}\left(\zeta_{\frac{N}{4}}^{2} \zeta_{-\frac{3 N}{4}}^{2}+\zeta_{\frac{3 N}{4}}^{2} \zeta_{-\frac{N}{4}}^{2}\right)=J_{\frac{N}{4}}^{2}-M_{\frac{N}{4}}^{2} .
$$

Theorem 1.4 now follows from the formulas (48), (56), and (57). 


\section{Proof of Theorem 1.3.}

To analyze the Hessian $Q_{\alpha, \beta}$ of (48) at $I=0$ we repeatedly encounter matrices of the form $E+\operatorname{diag}\left(\mu_{1}, \ldots, \mu_{N-1}\right)$, where $E$ is the $(N-1) \times(N-1)$-matrix

$$
E:=\left(\begin{array}{ccc}
1 & \ldots & 1 \\
\vdots & & \vdots \\
1 & \ldots & 1
\end{array}\right)
$$

and $\left(\mu_{k}\right)_{1 \leq k \leq N-1}$ are given complex numbers. The determinant of the matrix $E+\operatorname{diag}\left(\mu_{1}, \ldots, \mu_{N-1}\right)$ can be explicitly computed.

Lemma 6.1. Let $\left(\mu_{k}\right)_{1 \leq k \leq N-1}$ be given nonzero complex numbers. Then

$$
\operatorname{det}\left(E+\operatorname{diag}\left(\mu_{1}, \ldots, \mu_{N-1}\right)\right)=\left(1+\sum_{k=1}^{N-1} \frac{1}{\mu_{k}}\right) \cdot \prod_{k=1}^{N-1} \mu_{k} .
$$

In particular, $E+\operatorname{diag}\left(\mu_{1}, \ldots, \mu_{N-1}\right)$ is regular if and only if $\sum_{k=1}^{N-1} \frac{1}{\mu_{k}} \neq-1$.

Proof. Expanding $\operatorname{det}\left(E+\operatorname{diag}\left(\mu_{1}, \ldots, \mu_{N-1}\right)\right)$ with respect to its rows it follows that

$$
\operatorname{det}\left(E+\operatorname{diag}\left(\mu_{1}, \ldots, \mu_{N-1}\right)\right)=\prod_{k=1}^{N-1} \mu_{k}+\sum_{k=1}^{N-1} \prod_{l \neq k} \mu_{l}
$$

This leads to formula (59).

First let us treat the case $\alpha=0, \beta \neq 0$. The following proposition improves earlier results of Rink [13].

Proposition 6.2. Let $N$ be odd and assume that $\alpha=0$ in (2). Then the following holds:

(i) The Birkhoff normal form of $H_{V}$ up to order 4 is given by $\frac{N P^{2}}{2}+H_{0, \beta}(I)$, where

$$
H_{0, \beta}(I)=2 \sum_{k=1}^{N-1} \lambda_{k}^{2} I_{k}+\frac{\beta}{4 N}\left(\sum_{k=1}^{N-1} \lambda_{k}^{4} I_{k}^{2}+2 \sum_{1 \leq l \neq m \leq N-1} \lambda_{l}^{2} \lambda_{m}^{2} I_{l} I_{m}\right) .
$$

(ii) For any $\beta \neq 0, H_{0, \beta}(I)$ is nondegenerate at $I=0$.

Proof. The Birkhoff normal form (60) of $H_{V}$ is given by the formula (48) evaluated at $\alpha=0$. To investigate the Hessian $Q_{0, \beta}$ of $H_{0, \beta}(I)$ at $I=0$ we write

$$
Q_{0, \beta}=\frac{\beta}{4 N} \Delta P \Delta
$$

where

$$
\Delta=\operatorname{diag}\left(\sin \frac{k \pi}{N}\right)_{1 \leq k \leq N-1}
$$


and

$$
P=2 \cdot\left(E-\frac{1}{2} \operatorname{Id}_{N-1}\right)
$$

In view of (59) it follows that

$$
\operatorname{det} Q_{0, \beta}=\left(\frac{\beta}{4 N}\right)^{N-1} \cdot \operatorname{det} P \cdot \prod_{k=1}^{N-1} \sin ^{2} \frac{k \pi}{N},
$$

where by Lemma 6.1,

$$
\operatorname{det} P=2^{N-1}(1-2(N-1))(-1 / 2)^{N-1}=(-1)^{N}(2 N-3) \neq 0 .
$$

Hence, if $\beta \neq 0$, det $Q_{0, \beta} \neq 0$, and the nondegeneracy of $H_{0, \beta}(I)$ at $I=0$ follows.

Lemma 6.3. If $\beta<0$, then $Q_{0, \beta}$ has one negative eigenvalue, whereas if $\beta>0$, then $Q_{0, \beta}$ has $N-2$ negative eigenvalues. In particular, for any $\beta \neq 0, Q_{0, \beta}$ is indefinite (and $H_{0, \beta}$ is therefore not convex).

Proof. We want to use the decomposition (61) of $Q_{0, \beta}$ to show that $Q_{0, \beta}$ can be deformed continuously to $\frac{\beta}{4 N} P$ : Consider for $0 \leq t \leq 1$,

$$
Q_{0, \beta}(t):=\frac{\beta}{4 N}(t \Delta+(1-t) \mathrm{Id}) P(t \Delta+(1-t) \mathrm{Id}) .
$$

As $t \Delta+(1-t)$ Id is positive definite for any $0 \leq t \leq 1$ and $P$ is regular, $Q_{0, \beta}(t)$ is a symmetric regular $(N-1) \times(N-1)$-matrix for any $0 \leq t \leq 1$. For $t=0$, $Q_{0, \beta}(0)=\frac{\beta}{4 N} P$, whereas for $t=1, Q_{0, \beta}(1)=Q_{0, \beta}$. Therefore, index $\left(Q_{0, \beta}\right)$ (i.e. the number of negative eigenvalues of $\left.Q_{0, \beta}\right)$ coincides with index $\left(\frac{\beta}{4 N} P\right)$. The eigenvalues of $P$ are $\mu_{1}=2 N-3$ with multiplicity one and $\mu_{2}=-1$ with multiplicity $N-2$.

We now turn to the case $\alpha \neq 0$.

Proposition 6.4. Assume that $N$ is odd and $\alpha \neq 0$ in (2). Then, for $\alpha$ fixed, det $Q_{\alpha, \beta}$ is a polynomial in $\beta$ of degree $N-1$ and has $N-1$ real zeroes (counted with multiplicities) which we list in increasing order and denote by $\beta_{k}=\beta_{k}(\alpha)(1 \leq k \leq N-1)$. They satisfy $0<\beta_{1}<\alpha^{2}, 2 \alpha^{2}<\beta_{2} \leq \cdots \leq \beta_{N-1}$ and contain the $\llcorner(N-1) / 2\lrcorner$ distinct numbers

$$
\alpha^{2}\left(1+\left(\sin ^{2} \frac{k \pi}{N}\right)^{-1}\right) \quad\left(1 \leq k \leq\left\llcorner\frac{N-1}{2}\right\lrcorner\right)
$$

Moreover

$$
\operatorname{index}\left(Q_{\alpha, \beta}\right)= \begin{cases}1 & \text { for } \beta<\beta_{1} \\ 0 & \text { for } \beta_{1}<\beta<\beta_{2} \\ N-2 & \text { for } \beta>\beta_{N-1}\end{cases}
$$


Proof. Fix $\alpha \in \mathbb{R} \backslash\{0\}$ and consider the map $\beta \mapsto \operatorname{det}\left(Q_{\alpha, \beta}\right)$. It follows from (48) that $\operatorname{det}\left(Q_{\alpha, \beta}\right)$ is a polynomial in $\beta$ of degree at most $N-1$,

$$
\operatorname{det}\left(Q_{\alpha, \beta}\right)=\sum_{j=0}^{N-1} q_{j} \beta^{j}
$$

where $q_{0}=\operatorname{det}\left(Q_{\alpha, 0}\right)$ and $q_{N-1}=\operatorname{det}\left(Q_{0,1}\right)$. By Proposition 6.2, $\operatorname{det}\left(Q_{0,1}\right) \neq 0$, hence the degree of the polynomial $\operatorname{det}\left(Q_{\alpha, \beta}\right)$ is $N-1$. We claim that $\operatorname{det}\left(Q_{\alpha, \beta}\right)$ has $N-1$ real zeroes (counted with multiplicities). For $|\beta|$ large enough, index $\left(Q_{\alpha, \beta}\right)$ is equal to index $\left(Q_{0, \beta}\right)$. By Lemma 6.3, index $\left(Q_{0, \beta}\right)$ is $N-2$ for $\beta>0$ and 1 for $\beta<0$. Hence there exists $R>0$ such that index $\left(Q_{\alpha, \beta}\right)=N-2$ for any $\beta>R$ and $\operatorname{index}\left(Q_{\alpha, \beta}\right)=1$ for any $\beta<-R$. For $\beta=\alpha^{2}, Q_{\alpha, \alpha^{2}}$ is a positive multiple of the identity matrix, hence index $\left(Q_{\alpha, \alpha^{2}}\right)=0$. It then follows that index $\left(Q_{\alpha, \beta}\right)$ must change at least once in the open interval $\left(-\infty, \alpha^{2}\right)$ and at least $N-2$ times (counted with multiplicities) in $\left(\alpha^{2}, \infty\right)$. Since a change of index $\left(Q_{\alpha, \beta}\right)$ induces a zero of $\operatorname{det}\left(Q_{\alpha, \beta}\right)$ (counted with multiplicities), our consideration shows that $\beta \mapsto \operatorname{det}\left(Q_{\alpha, \beta}\right)$ has $N-1$ real zeroes. Further we have $\beta_{1}(\alpha)<\alpha^{2}<\beta_{2}(\alpha)$.

Next we prove that $\beta_{1}(\alpha)>0$, i.e. that $Q_{\alpha, \beta}$ is regular for any $\beta \leq 0$. Write $Q_{\alpha, \beta}$ as a product,

$$
Q_{\alpha, \beta}=\frac{\alpha^{2}-\beta}{4 N} \Delta P_{\alpha, \beta} \Delta,
$$

where $\Delta$ is given by (62) and $P_{\alpha, \beta}$ is given by

$$
P_{\alpha, \beta}=-2\left(E+\operatorname{diag}\left(-\frac{1}{2}\left(1+\frac{\gamma(\alpha, \beta)}{\sin ^{2} \frac{k \pi}{N}}\right)\right)_{1 \leq k \leq N-1}\right),
$$

where $E$ is given by (58) and

$$
\gamma(\alpha, \beta):=\frac{\alpha^{2}}{\alpha^{2}-\beta}
$$

As $-\infty<\beta \leq 0$ it follows that $0<\gamma(\alpha, \beta) \leq 1$ and $-\frac{1}{2}\left(1+\frac{\gamma(\alpha, \beta)}{\sin ^{2} \frac{k \pi}{N}}\right)<0$ for any $1 \leq k \leq N-1$. Lemma 6.1 says that $P_{\alpha, \beta}$ is regular if $f(\gamma(\alpha, \beta)) \neq 0$, where

$$
f(\gamma):=1-2 \sum_{k=1}^{N-1}\left(1+\gamma / \sin ^{2} \frac{k \pi}{N}\right)^{-1}
$$

in the interval $0<\gamma \leq 1$. Note that $f(\gamma)$ is increasing in $0<\gamma \leq 1$ and $f(1)$ can be estimated as follows. Using that $N$ is assumed to be odd one has

$$
\begin{gathered}
f(1)=1-4 \sum_{k=1}^{\frac{N-1}{2}} \frac{\sin ^{2} \frac{k \pi}{N}}{1+\sin ^{2} \frac{k \pi}{N}}<1-4 \frac{\sin ^{2} \frac{(N-1) \pi}{2 N}}{1+\sin ^{2} \frac{(N-1) \pi}{2 N}} \\
=1-4 \frac{\cos ^{2} \frac{\pi}{2 N}}{1+\cos ^{2} \frac{\pi}{2 N}}=-3+\frac{4}{1+\cos ^{2} \frac{\pi}{2 N}} .
\end{gathered}
$$


As for $N \geq 3$,

$$
-3+\frac{4}{1+\cos ^{2} \frac{\pi}{2 N}}<-3+\frac{4}{1+\cos ^{2} \frac{\pi}{6}}=-\frac{5}{7},
$$

we conclude that $f(1)<0$. Hence we have shown that $f(\gamma)<0$ for $0<\gamma \leq 1$, and therefore $P_{\alpha, \beta}$ is regular for $\beta \leq 0$ by Lemma 6.1. Hence we have proved that $0<\beta_{1}(\alpha)$.

By the same method we prove that $\beta_{2}(\alpha)>2 \alpha^{2}$, or equivalently, since we have already shown that $\beta_{2}(\alpha)>\alpha^{2}$, that $Q_{\alpha, \beta}$ is regular for any $\alpha^{2}<\beta \leq 2 \alpha^{2}$. We decompose $Q_{\alpha, \beta}$ as in (63) and (64), and according to the definition (65) of $\gamma(\alpha, \beta)$, $\alpha^{2}<\beta \leq 2 \alpha^{2}$ corresponds to $\gamma(\alpha, \beta) \leq-1$. For such $\gamma$ 's, we have $1+\gamma / \sin ^{2} \frac{k \pi}{N}<0$, and hence $-\frac{1}{2}\left(1+\gamma / \sin ^{2} \frac{k \pi}{N}\right)>0$ for any $1 \leq k \leq N-1$. Moreover, it also follows that $\left(1+\gamma / \sin ^{2} \frac{k \pi}{N}\right)^{-1}<0$, which allows us to conclude that

$$
f(\gamma)=1-2 \sum_{k=1}^{N-1}\left(1+\gamma / \sin ^{2} \frac{k \pi}{N}\right)^{-1}>1>0 .
$$

According to Lemma 6.1, this proves the regularity of $P_{\alpha, \beta}$ and hence of $Q_{\alpha, \beta}$ for $\alpha^{2}<\beta \leq 2 \alpha^{2}$.

Finally introduce $\mu_{k}:=-\frac{1}{2}\left(1+\gamma(\alpha, \beta) / \sin ^{2} \frac{k \pi}{N}\right)$ and note that for $\beta$ with $\gamma(\alpha, \beta)=$ $-\sin ^{2} \frac{k_{0} \pi}{N}$ for some $1 \leq k_{0} \leq\left\llcorner\frac{N-1}{2}\right\lrcorner$ one has $\mu_{k_{0}}=\mu_{N-k_{0}}=0$. As $k_{0} \neq N-k_{0}$ if $1 \leq k_{0} \leq\llcorner(N-1) / 2\lrcorner$, it then follows that $P_{\alpha, \beta}$ has two equal rows and is therefore singular. Note that $\gamma(\alpha, \beta)=-\sin ^{2} \frac{k_{0} \pi}{N}$ corresponds to $\beta=\alpha^{2}\left(1+\sin ^{-2} \frac{k_{0} \pi}{N}\right)$ and we have proved that $\beta \mapsto \operatorname{det}\left(Q_{\alpha, \beta}\right)$ has at least $\llcorner(N-1) / 2\lrcorner$ different zeroes in the interval $\left(\alpha^{2}, \infty\right)$. The statement about index $\left(Q_{\alpha, \beta}\right)$ easily follows from the above analysis.

Proof of Theorem 1.3. Part (i) is proved by Proposition 6.4, whereas (ii) follows from Proposition 6.2 and Lemma 6.3.

\section{A. Proof of Lemma 4.3}

For the convenience of the reader, we provide a detailed proof of Lemma 4.3 in this appendix. This lemma and its proof are due to Beukers and Rink - see ([13], Appendix A). Recall that $K_{4} \backslash K_{4}^{N} \subseteq \mathbb{Z}^{4}$ denotes the subset of quadruples $\left(k_{1}, k_{2}, k_{3}, k_{4}\right)$ satisfying $1 \leq\left|k_{i}\right| \leq N-1(1 \leq i \leq 4)$ and $k_{1}+k_{2}+k_{3}+k_{4} \equiv 0 \bmod N$ so that there are no integers $l, m$ with $\{l, m,-l,-m\}=\left\{k_{1}, k_{2}, k_{3}, k_{4}\right\}$, and

$$
K_{4}^{r e s}:=K_{r e s}^{+} \cup K_{r e s}^{-} \subseteq K_{4},
$$

where

$$
\begin{aligned}
K_{\text {res }}^{ \pm}:= & \left\{\left(k_{1}, k_{2}, k_{3}, k_{4}\right) \in K_{4} \mid \exists l \in \mathbb{N}: 1 \leq l \leq \frac{N}{4}\right. \text { so that } \\
& \left.\left\{k_{1}, k_{2}, k_{3}, k_{4}\right\}=\left\{ \pm l, \pm l \mp N, \frac{N}{2} \mp l,-\frac{N}{2} \mp l\right\}\right\} .
\end{aligned}
$$

Note that $K_{4}^{\text {res }}=\emptyset$ if $N$ is odd. Let us restate Lemma 4.3 as follows: 
Lemma A.1. ([13]). Let $\left(k_{1}, k_{2}, k_{3}, k_{4}\right)$ be an element of $K_{4} \backslash K_{4}^{N}$. Then $\left(k_{1}, k_{2}, k_{3}, k_{4}\right) \in$ $K_{4}^{\text {res }}$ if and only if

$$
\sin \frac{k_{1} \pi}{N}+\sin \frac{k_{2} \pi}{N}+\sin \frac{k_{3} \pi}{N}+\sin \frac{k_{4} \pi}{N}=0 .
$$

Let us make a few preparations for the proof of Lemma A.1. By a straightforward computation one sees that the "only if"-part of the claimed equivalence holds:

Lemma A.2. For any $\left(k_{1}, k_{2}, k_{3}, k_{4}\right) \in K_{4}^{\text {res }}$, one has $\sum_{i=1}^{4} \sin \frac{k_{i} \pi}{N}=0$.

So it remains to prove the converse. First we consider some special cases.

Lemma A.3. Let $\left(k_{1}, k_{2}, k_{3}, k_{4}\right) \in K_{4} \backslash\left(K_{4}^{N} \cup K_{4}^{\text {res }}\right)$. If there exist $l, m, n \in \mathbb{Z}$ such that

(i) $\left\{k_{1}, k_{2}, k_{3}, k_{4}\right\}=\{l,-l, m, n\}$, or

(ii) $\left\{k_{1}, k_{2}, k_{3}, k_{4}\right\}=\{l, N-l, m, n\}$ with $1 \leq l \leq N-1$, or

(iii) $\left\{k_{1}, k_{2}, k_{3}, k_{4}\right\}=\{l,-N-l, m, n\}$ with $-(N-1) \leq l \leq-1$,

then

$$
\sum_{i=1}^{4} \sin \frac{k_{i} \pi}{N} \neq 0
$$

Proof. In case (i), it follows that $m+n=N$ (and thus $1 \leq m, n \leq N-1$ ) or $m+n=-N$ (and thus $-(N-1) \leq m, n \leq-1)$. Hence in both cases, $\sin \frac{m \pi}{N}$ and $\sin \frac{n \pi}{N}$ have the same sign and $\sum_{i=1}^{4} \sin \frac{k_{i} \pi}{N}=\sin \frac{m \pi}{N}+\sin \frac{n \pi}{N} \neq 0$. In the case (ii), by assumption, $m+n \equiv 0 \bmod N$. The case $m+n=0$ has already been treated under (i). If $m+n=N$, then $\sin \frac{k_{i} \pi}{N}>0$ for any $1 \leq i \leq 4$. If $m+n=-N$, then $m<0$, and $m \notin\{-l,-N+l\}$. Thus $n=-N-m<0$, and therefore $\sum_{i=1}^{4} \sin \frac{k_{i} \pi}{N}=2 \sin \frac{l \pi}{N}-2 \sin \frac{(-m) \pi}{N} \neq 0$. The case (iii) is treated similarly as (ii).

Another special case is treated in the following lemma.

Lemma A.4. Assume that $\left(k_{1}, k_{2}, k_{3}, k_{4}\right) \in K_{4} \backslash K_{4}^{N}$ satisfies

$$
k_{i}+k_{j} \not \equiv 0 \bmod N \quad \forall 1 \leq i, j \leq 4 .
$$

If there exist $l, n \in\left\{k_{1}, k_{2}, k_{3}, k_{4}\right\}$ with

$$
\sin \frac{l \pi}{N}+\sin \frac{n \pi}{N}=0
$$

then

$$
\sum_{i=1}^{4} \sin \frac{k_{i} \pi}{N}=0
$$

implies that $\left(k_{1}, k_{2}, k_{3}, k_{4}\right) \in K_{4}^{r e s}$. 
Proof. From the assumptions (66)-(67) it follows that there exists $1 \leq l \leq N-1$ so that $\left\{k_{1}, k_{2}, k_{3}, k_{4}\right\}=\{l,-N+l, m, n\}$ for some $m, n \in \mathbb{Z}$. Then $\sin \frac{l \pi}{N}+\sin \frac{(-N+l) \pi}{N}=0$, and hence by (68), $\sin \frac{m \pi}{N}+\sin \frac{n \pi}{N}=0$. W.1.o.g. assume that $1 \leq m \leq N-1$. Then either $n=-m$ or $n=-N+m$. If $n=-m$, then $\left(k_{1}, k_{2}, k_{3}, k_{4}\right) \in K_{4}^{r e s}$ by Lemma A.3 (i). If $n=-N+m$, then one has

$$
\sum_{i=1}^{4} k_{i}=2 l-N+2 m-N=2(l+m)-2 N .
$$

Note that $2(l+m)-2 N$ cannot be an even multiple of $N$, as otherwise $l+m \equiv 0 \bmod$ $N$, violating (66). If, in addition, $N$ is odd, then $2(l+m)-2 N$ cannot be odd multiple of $N$. Hence in the case $N$ is odd we conclude that $\sum_{i=1}^{4} k_{i} \not \equiv 0 \bmod N$, contradicting the assumption $\left(k_{1}, k_{2}, k_{3}, k_{4}\right) \in K_{4}$.

If $N$ is even, it is however possible that $2(l+m)-2 N$ equals $\pm N$ : If $2(l+m)-2 N=N$, i.e. $l+m=\frac{3}{2} N$, it follows that $\frac{N}{2}<l, m \leq N-1$, and $\left(k_{1}, k_{2}, k_{3}, k_{4}\right) \in K_{\text {res }}^{-}$as $\left\{k_{1}, k_{2}, k_{3}, k_{4}\right\}=\left\{-l^{\prime},-l^{\prime}+N, \frac{N}{2}+l^{\prime},-\frac{N}{2}+l^{\prime}\right\}$ with $l^{\prime}=l-\frac{N}{2}$. If $2(l+m)-2 N=-N$, i.e. $l+m=\frac{N}{2}$, it follows similarly that $\left(k_{1}, k_{2}, k_{3}, k_{4}\right) \in K_{r e s}^{+}$as $\left\{k_{1}, k_{2}, k_{3}, k_{4}\right\}=$ $\left\{l, l-N, \frac{N}{2}-l,-\frac{N}{2}-l\right\}$. So in both cases, we conclude that $\left(k_{1}, k_{2}, k_{3}, k_{4}\right) \in K_{4}^{r e s}$.

In view of Lemma A.3 and Lemma A.4 in order to prove Lemma A.1 it remains to show the following

Lemma A.5. Assume that $\left(k_{1}, k_{2}, k_{3}, k_{4}\right) \in K_{4}$ satisfies (66). Iffor any $1 \leq i, j \leq 4$,

$$
\sin \frac{k_{i} \pi}{N}+\sin \frac{k_{j} \pi}{N} \neq 0
$$

(and thus $\left(k_{1}, k_{2}, k_{3}, k_{4}\right) \notin K_{4}^{N} \cup K_{4}^{\text {res }}$ ), then

$$
\sum_{i=1}^{4} \sin \frac{k_{i} \pi}{N} \neq 0
$$

To prove Lemma A.5 let us first rewrite (68), using Euler's formula for the sine function,

$$
\sum_{1 \leq|j| \leq 4} \zeta_{j}=0
$$

where $\zeta_{ \pm j}= \pm e^{ \pm i k_{j} \pi / N}$ are $2 N^{\text {th }}$ roots of unity. Note that for any quadruple $\left(k_{1}, k_{2}, k_{3}\right.$, $\left.k_{4}\right) \in K_{4} \backslash K_{4}^{N}$ satisfying (69) one has

$$
\zeta_{j}+\zeta_{j^{\prime}} \neq 0 \quad \forall 1 \leq|j| \leq\left|j^{\prime}\right| \leq 4
$$

Indeed for any $1 \leq|j| \leq\left|j^{\prime}\right| \leq 4$ one has $\operatorname{Im} \zeta_{j}+\operatorname{Im} \zeta_{j^{\prime}}=\sin \frac{k_{|j|} \pi}{N}+\sin \frac{k_{\left|j^{\prime}\right|} \pi}{N}$ which does not vanish by assumption (69).

Let us first discuss Eq. (70) and its solutions in general, i.e. we consider the equation

$$
\zeta_{1}+\cdots+\zeta_{8}=0
$$

and want to study its solutions, $\left(\zeta_{l}\right)_{1 \leq l \leq 8}$, on the unit circle $S^{1}:=\{z \in \mathbb{C}|| z \mid=1\}$. 
We need an auxiliary result which we discuss first. Let $n \geq 2$ be arbitrary and assume that the sequence $\left(\zeta_{i}\right)_{1 \leq i \leq n} \subseteq S^{1}$ has no vanishing subsums (i.e. $\sum_{l \in J} \zeta_{l} \neq 0$ for any $\emptyset \neq J \subsetneq\{1, \ldots, n\})$ and satisfies the equation

$$
\sum_{i=1}^{n} \zeta_{i}=0
$$

Let $M \in \mathbb{N}$ be the smallest integer with the property that $\left(\zeta_{i} / \zeta_{j}\right)^{M}=1$ for all $1 \leq i$, $j \leq n$. Then there exists $\xi \in S^{1}$ so that $\zeta_{i}^{M}=\xi^{M}$ for any $1 \leq i \leq n$. W.1.o.g. we can assume that $\xi=1$. Furthermore, let $p^{k}$ be a prime power dividing $M$ so that $M / p^{k}$ and $p$ are relatively prime and define

$$
M^{\prime}=: M / p \text { and } \eta:=e^{2 \pi i / p^{k}} .
$$

Then for any $1 \leq l \leq n$ there exists a unique integer $0 \leq \mu(l) \leq p-1$ such that $\zeta_{l}=\tilde{\zeta}_{l} \cdot \eta^{\mu(l)}$, where $\bar{\zeta}_{l}$ is an element of the field $K:=\mathbb{Q}\left(e^{2 \pi i / M^{\prime}}\right)$. (As $\zeta_{l}^{M}=1$ there exists $0 \leq r_{l} \leq M-1$ with $\zeta_{l}=e^{\frac{2 \pi i}{M} r_{l}}$. If $r_{l} \equiv 0 \bmod p$ choose $\mu(l)=0$. If $r_{l} \not \equiv 0 \bmod$ $p$ choose $1 \leq \mu(l) \leq p-1$ so that $r_{l} \equiv \frac{M}{p^{k}} \mu(l) \bmod p$.) Hence (72) can be written as

$$
0=\sum_{l=1}^{n} \zeta_{l}=\sum_{s=0}^{p-1}\left(\sum_{l \in \mu^{-1}(s)} \zeta_{l}\right)=\sum_{s=0}^{p-1}\left(\sum_{l \in \mu^{-1}(s)} \tilde{\zeta}_{l}\right) \eta^{s}
$$

We need the following algebraic fact (see e.g. [17], §60-61):

Proposition A.6. The minimal polynomial of $\eta=e^{2 \pi i / p^{k}}$ over the field $K=\mathbb{Q}\left(e^{2 \pi i / M^{\prime}}\right)$ is given by $X^{p}-\eta^{p}$ if $k \geq 2$ and $X^{p-1}+X^{p-2}+\cdots+X+1$ if $k=1$.

We now claim that $M$ is square-free, or equivalently that for any prime power $p^{k}$ divi$\operatorname{ding} M$,

$$
k=1 \text {. }
$$

Indeed, Eq. (74) shows that the minimal polynomial of $\zeta$ has degree at most $p-1$, which by Proposition A.6 is only satisfied in the case $k=1$.

Further we claim that there exists $\sigma \in \mathbb{C} \backslash\{0\}$ so that

$$
\sum_{l \in \mu^{-1}(s)} \tilde{\zeta}_{l}=\sigma \quad \forall 0 \leq s \leq p-1 .
$$

The existence of such a $\sigma$ follows from Proposition A.6: As $k=1$ by (75), the minimal polynomial of $\eta$ over $K$ is given by $X^{p-1}+X^{p-2}+\cdots+X+1$. Since this is a polynomial of degree $p-1$ the polynomial on the right-hand side of (74) must be a scalar multiple of the minimal polynomial. Hence all the coefficients $\sum_{l \in \mu^{-1}(s)} \tilde{\zeta}_{l}$ have the same value $\sigma \in \mathbb{C}$. As $\sum_{l \in \mu^{-1}(s)} \zeta_{l}=\sigma \eta^{s}$, the additional property $\sigma \neq 0$ follows from the assumption that there are no vanishing subsums. Hence we can assume w.l.o.g. that $\sigma=1$.

Next we claim that

$$
p \leq n
$$


In other words, possible prime factors of $M$ are bounded by the number of summands in (72). To prove (77), note that it follows from (76) that for any $0 \leq s \leq p-1$ there exists $1 \leq l \leq n$ such that $\mu(l)=s$, i.e. the map $\mu:\{1, \ldots, n\} \rightarrow\{0, \ldots, p-1\}$ is onto. This establishes (77).

The map $\mu$ induces the partition $\left(\sharp \mu^{-1}(s)\right)_{0 \leq s \leq p-1}$ of the positive integer $n$ into $p$ summands,

$$
n=\sum_{s=0}^{p-1} \sharp \mu^{-1}(s) .
$$

Lemma A.7. ([13], Appendix A). For any solution $\left\{\zeta_{1}, \ldots, \zeta_{8}\right\}$ of (71) contained in $S^{1}$ without vanishing subsums there exists $\xi \in S^{1}$ such that either

$$
\left\{\zeta_{1}, \ldots, \zeta_{8}\right\}=\left\{-\xi \alpha,-\xi \alpha^{2}\right\} \cup\left\{\xi \gamma^{j} \mid 1 \leq j \leq 6\right\}
$$

or

$$
\left\{\zeta_{1}, \ldots, \zeta_{8}\right\}=\left\{-\xi \alpha^{l},-\xi \alpha^{l} \cdot \beta^{i},-\xi \alpha^{l} \cdot \beta^{j} \mid 1 \leq l \leq 2\right\} \cup\left\{\xi \beta^{k}, \xi \beta^{m}\right\},
$$

where the quadruple $(i, j, k, m)$ is a permutation of $(1,2,3,4)$ and

$$
\alpha:=e^{\frac{2 \pi i}{3}}, \quad \beta:=e^{\frac{2 \pi i}{5}}, \quad \gamma:=e^{\frac{2 \pi i}{7}} .
$$

Proof. By a straightforward computation one verifies that the sets of the form (79) or (80) satisfy (71). It remains to prove that these are the only solutions of (71) of this type.

We classify the solutions of (71) according to the possible values of $p$, which we now assume to be the largest prime dividing $M$. Since $n=8$, by (77), the possible values of $p$ are 2, 3, 5, and 7. If $p=2$, then, by (75), $M=2$ and therefore there exists $\xi \in S^{1}$ so that $\zeta_{j}= \pm \xi$ for any $1 \leq j \leq n$. In this case there exists a solution of (72) without vanishing subsums only if $n=2$. (In this case, they are given by $\left\{\zeta_{1}, \zeta_{2}\right\}=\xi\{1,-1\}$ with $\xi \in S^{1}$.) If $p=3$, then $M=3$ or $M=3 \cdot 2$, and there exists a solution of (72) without vanishing subsums only if $n=3$. (In this case, they are given by $\left\{\zeta_{1}, \zeta_{2}, \zeta_{3}\right\}=\xi\left\{1, \alpha, \alpha^{2}\right\}$ with $\xi \in S^{1}$.) If $p=5$, then $\eta=\beta$ in (73). Up to permutations, there are the following three partitions of 8 into 5 summands, $(4,1,1,1,1),(3,2,1,1,1)$, and $(2,2,2,1,1)$. In a straightforward way one shows that the partitions $(4,1,1,1,1)$ and $(3,2,1,1,1)$ and their permutations give rise to solutions of Eq. (71) with vanishing subsums. E.g. the solutions corresponding to $(4,1,1,1,1)$ are given by $\xi \cdot\left(-\beta,-\beta^{2},-\beta^{3},-\beta^{4}, \beta, \beta^{2}, \beta^{3}, \beta^{4}\right)$ with $\xi \in S^{1}$, whereas the solutions corresponding to $(3,2,1,1,1)$ are $\xi \cdot\left(-i, 1, i,-\alpha \beta,-\alpha^{2} \beta, \beta^{2}, \beta^{3}, \beta^{4}\right)$ with $\xi \in S^{1}$. On the other hand the partition $(2,2,2,1,1)$ leads to the solutions

$$
\left(\zeta_{1}, \ldots, \zeta_{8}\right)=\xi\left(-\alpha,-\alpha^{2},-\alpha \beta,-\alpha^{2} \beta,-\alpha \beta^{2},-\alpha^{2} \beta^{2}, \beta^{3}, \beta^{4}\right)
$$

with $\xi \in S^{1}$. They are the solutions (80) with $(i, j, k, m)=(1,2,3,4)$. Permutations of the partition $(2,2,2,1,1)$ again lead to solutions of the type $(80)$, but with $(i, j, k, m)$ given by a permutation of $(1,2,3,4)$.

If $p=7$, then $\eta=\gamma$ in (73). Then, up to permutations, $(2,1,1,1,1,1,1)$ is the only possible partition of 8 into 7 summands. The partition $(2,1,1,1,1,1,1)$ leads to the solutions

$$
\left(\zeta_{1}, \ldots, \zeta_{8}\right)=\xi\left(-\alpha,-\alpha^{2}, \gamma, \ldots, \gamma^{6}\right)
$$

with $\xi \in S^{1}$, where we used that $1=-\alpha-\alpha^{2}$. They are of type (79). Any permutation of $(2,1,1,1,1,1,1)$ leads to the same kind of solutions. 
Lemma A.8. ([13], Appendix A). For any solution $\left\{\zeta_{1}, \ldots, \zeta_{8}\right\}$ of (71) contained in $S^{1}$ without vanishing subsums of length 2 but having a vanishing subsum of length 3, 4, or 5 , there exist $\xi, \xi^{\prime} \in S^{1}$ such that

$$
\left\{\zeta_{1}, \ldots, \zeta_{8}\right\}=\left\{\xi \alpha^{l} \mid 0 \leq l \leq 2\right\} \cup\left\{\xi^{\prime} \beta^{m} \mid 0 \leq m \leq 4\right\},
$$

where again $\alpha=e^{2 \pi i / 3}$ and $\beta=e^{2 \pi i / 5}$.

Proof. Again, one verifies by a direct computation that the solutions (81) of (71) have the desired properties. It remains to prove that they are the only ones. First note that under the hypotheses of the lemma, vanishing subsums of length 4 cannot occur, since the latter ones would imply the existence of vanishing subsums of length 2 , which by assumption is excluded. Hence, in order to find solutions of (72) for $n=8$ with the desired properties, we have to find all solutions of (72) without vanishing subsums for $n=3$ and $n=5$. Note that by (77), $p=n$ for $n=3$ or $n=5$. By the considerations in the proof of Lemma A.7, the former ones are given by $\left(\zeta_{1}, \zeta_{2}, \zeta_{3}\right)=\xi\left(1, \alpha, \alpha^{2}\right)$ and the latter ones by $\left(\zeta_{1}, \ldots, \zeta_{5}\right)=\xi^{\prime}\left(1, \beta, \beta^{2}, \beta^{3}, \beta^{4}\right)$ with $\xi, \xi^{\prime} \in S^{1}$. This proves the lemma.

We are now ready to prove Lemma A.5.

Proof of Lemma A.5. We first select from (79), (80) and (81) all the solutions $\left(\zeta_{1}, \ldots, \zeta_{8}\right)$ of (71) which are of the form (68) (after multiplication by $2 i$ ). This amounts to selecting the solutions $\left(\zeta_{1}, \ldots, \zeta_{8}\right)$ of (71) having the property that $\left\{\zeta_{1}, \ldots, \zeta_{8}\right\}$ is invariant under the map $\zeta \mapsto-\zeta^{-1}$. It requires to choose $\xi$ and $\xi^{\prime}$ in (79), (80), and (81) appropriately. Let us explain this procedure in detail for the solutions of type (79).

First we rewrite the solution (79),

$$
\left(\zeta_{1}, \ldots, \zeta_{8}\right)=\xi \cdot\left(-\alpha,-\alpha^{2}, \gamma, \gamma^{2}, \gamma^{3}, \gamma^{4}, \gamma^{5}, \gamma^{6}\right)=e^{\frac{2 \pi i x}{42}}\left(e^{\frac{2 \pi i t_{k}}{42}}\right)_{1 \leq k \leq 8},
$$

where $\xi=e^{2 \pi i x / 42}$ with $x \in \mathbb{R} / 42 \mathbb{Z}$ and

$$
\left(t_{1}, \ldots, t_{8}\right)=(6,7,12,18,24,30,35,36) .
$$

The required invariance of the set of the $\zeta_{k}$ 's under the map $\zeta \mapsto-\zeta^{-1}$ is equivalent to the invariance of the set of the $\left(t_{k}+x\right)$ 's under the map $t \mapsto 21-t(\bmod 42)$. Since the set (82) of the $t_{k}$ 's is invariant under the map $t \mapsto-t(\bmod 42),\left\{t_{k}+x \mid 1 \leq k \leq 8\right\}$ is invariant under $t \mapsto 21-t(\bmod 42)$, if we choose $x:=\frac{21}{2}$ or $\xi=i$. Then the equation $\sum_{i=1}^{8} \zeta_{i}=0$ reads

$$
e^{\frac{11 \pi i}{14}}+e^{\frac{5 \pi i}{6}}+e^{\frac{15 \pi i}{14}}+e^{\frac{19 \pi i}{14}}+e^{\frac{23 \pi i}{14}}+e^{\frac{27 \pi i}{14}}+e^{\frac{\pi i}{6}}+e^{\frac{3 \pi i}{14}}=0
$$

or $\sin \frac{\pi}{6}+\sin \frac{3 \pi}{14}+\sin \frac{15 \pi}{14}+\sin \frac{19 \pi}{14}=0$. Choosing all arguments in $(0, \pi)$, the latter identity reads

$$
\sin \frac{\pi}{6}+\sin \frac{3 \pi}{14}-\sin \frac{\pi}{14}-\sin \frac{5 \pi}{14}=0 .
$$

For the solutions of type (80), one gets

$$
\sin \frac{\pi}{6}+\sin \frac{13 \pi}{30}-\sin \frac{7 \pi}{30}-\sin \frac{3 \pi}{10}=0
$$


and

$$
\sin \frac{\pi}{6}+\sin \frac{\pi}{30}-\sin \frac{11 \pi}{30}+\sin \frac{\pi}{10}=0 .
$$

Let us briefly explain how (84)-(85) can be obtained. Note that from the 24 permutations of $(1,2,3,4)$ in $(80)$, there are only six which lead to different sets of the $\zeta_{i}$ 's, since interchanging $i$ and $j$ or $k$ and $m$ leaves the set on the right-hand side of (80) invariant. In the resulting six different cases, we again write $\left\{\zeta_{1}, \ldots, \zeta_{8}\right\}=\xi \cdot\left\{e^{2 \pi i \cdot \frac{t_{1}}{30}}, \ldots, e^{2 \pi i \cdot \frac{t_{8}}{30}}\right\}$ with $t_{i}$ in $\mathbb{R} / 30 \mathbb{Z}$. Then, up to translations, there are only two different types of solutions emerging from these six cases. With the appropriate choices of $\xi$, one gets the solutions (84) and (85).

Finally, for the solutions of type (81), one gets

$$
\sin \frac{\pi}{2}-\sin \frac{\pi}{6}+\sin \frac{\pi}{10}-\sin \frac{3 \pi}{10}=0
$$

The procedure to obtain (86) is basically the same as in the preceding cases. We write (81) as $\left\{\zeta_{1}, \ldots, \zeta_{8}\right\}=\xi \cdot\left\{\alpha^{l}, \lambda \cdot \beta^{m} \mid 0 \leq l \leq 2,0 \leq m \leq 4\right\}$ and first choose $\lambda \in S^{1}$ so that the set $\left\{\alpha^{l}, \lambda \cdot \beta^{m} \mid 0 \leq l \leq 2,0 \leq m \leq 4\right\}$ is symmetric with respect to some axis through the origin, and then choose $\xi$ so that this axis is the imaginary axis.

To finish the proof of Lemma A.5 we show that all the solutions $\left(k_{1}, k_{2}, k_{3}, k_{4}\right)$ of $\sum_{i=1}^{4} \sin \frac{k_{i} \pi}{N}=0$ obtained in (83)-(86) and the additional ones obtained by replacing $0<x<\pi$ in $\sin x$ by $\pi-x$ satisfy $\sum_{i=1}^{4} k_{i} \not \equiv 0 \bmod N$ and hence are not in $K_{4}$.

For the solutions obtained in (83)-(86), $N$ is even. Hence if $N$ is odd, then there is no quadruple $\left(k_{1}, k_{2}, k_{3}, k_{4}\right) \in K_{4}$ such that (68) and (69) are satisfied. This finishes the proof of Lemma A.5 in this case.

For the rest of the proof, we assume that $N$ is even. If $N=42 r$ for some $r \in \mathbb{N}$, (83) becomes

$$
\sin \frac{7 r \pi}{42 r}+\sin \frac{9 r \pi}{42 r}+\sin \frac{(-3 r) \pi}{42 r}+\sin \frac{(-15 r) \pi}{42 r}=0,
$$

and we have $7 r+9 r-3 r-15 r=-2 r \not \equiv 0 \bmod 42 r$. Hence the corresponding quadruple $\left(k_{1}, k_{2}, k_{3}, k_{4}\right)$ is not in $K_{4}$. For the quadruples obtained by replacing $0<x<\pi$ in $\sin x$ by $\pi-x$ in some of the summands in (83), the condition $\sum_{i=1}^{4} k_{i} \not \equiv 0 \bmod 42 r$ amounts to

$$
\pm 7 \pm 9 \pm 3 \pm 15 \not \equiv 0 \bmod 42
$$

for any combination of plus and minus signs. The relations (87) are easily verified. Similarly, one verifies that the quadruples $\left(k_{1}, k_{2}, k_{3}, k_{4}\right)$ satisfying (84), (85), or (86) are not in $K_{4}$ by showing that

$$
\pm 5 \pm 13 \pm 7 \pm 9 \not \equiv 0, \quad \pm 5 \pm 1 \pm 11 \pm 3 \not \equiv 0, \quad \pm 15 \pm 5 \pm 3 \pm 9 \not \equiv 0 \bmod 30,
$$

again for any combination of plus and minus signs. Hence we have shown that none of the solutions $\left(k_{1}, k_{2}, k_{3}, k_{4}\right)$ of (68) is an element of $K_{4}$. This completes the proof of Lemma A.5.

Proof of Lemma A.1. The claimed statement follows from Lemma A.2, A.3, A.4, and A.5. 


\section{B. Details of Section 3}

We begin by expressing the FPU Hamiltonian $H_{V}$ in relative coordinates. Introduce $\left(v=\left(v_{j}\right)_{1 \leq j \leq N-1}, v_{N}\right) \in \mathbb{R}^{N}$ given by (11). Then $\left(v, v_{N}\right)=M q$ is the linear change of the coordinates $q_{1}, \ldots, q_{N}$, where $M$ is given by

$$
M=\left(\begin{array}{ccccc}
-1 & 1 & 0 & \ldots & 0 \\
0 & \ddots & \ddots & & \vdots \\
\vdots & & & & 0 \\
0 & \ldots & 0 & -1 & 1 \\
N^{-1} & \ldots & & \ldots & N^{-1}
\end{array}\right)
$$

The variables $\left(u=\left(u_{j}\right)_{1 \leq j \leq N-1}, u_{N}\right) \in \mathbb{R}^{N}$ conjugate to $\left(v, v_{N}\right)$ are then given by $\left(M^{T}\right)^{-1} p$. The inverse of $M^{T},\left(M^{T}\right)^{-1}$, can be computed to be

$$
\left(M^{T}\right)^{-1}=\frac{1}{N}\left(\begin{array}{cccc}
1 & \ldots & \ldots & 1 \\
2 & \ldots & \ldots & 2 \\
\vdots & & & \vdots \\
\vdots & & & \vdots \\
N & \ldots & \ldots & N
\end{array}\right)-\left(\begin{array}{ccccc}
1 & 0 & \ldots & \ldots & 0 \\
1 & 1 & 0 & \ldots & 0 \\
\vdots & & & \vdots \\
1 & \ldots & & 1 & 0 \\
0 & \ldots & & \ldots & 0
\end{array}\right) .
$$
Hence

Note that by (89), $u_{k}=k P-\sum_{j=1}^{k} p_{j}$ for any $1 \leq k \leq N-1$ and $u_{N}=N P$.

$$
p_{1}=-u_{1}+P ; \quad p_{N}=u_{N-1}+P ; \quad p_{k}=\left(u_{k-1}-u_{k}\right)+P \quad(2 \leq k \leq N-1),
$$

and thus

$$
\frac{1}{2} \sum_{j=1}^{N} p_{j}^{2}=\frac{N P^{2}}{2}+\frac{1}{2}\left(u_{1}^{2}+\left(u_{1}-u_{2}\right)^{2}+\cdots+\left(u_{N-2}-u_{N-1}\right)^{2}+u_{N-1}^{2}\right) .
$$

Moreover, using that $q_{N+1}-q_{N}=q_{1}-q_{N}=-\sum_{k=1}^{N-1}\left(q_{k+1}-q_{k}\right)$ one gets for any $s \in \mathbb{Z}_{\geq 1}$,

$$
\sum_{j=1}^{N}\left(q_{j+1}-q_{j}\right)^{s}=\sum_{k=1}^{N-1} v_{k}^{s}+(-1)^{s}\left(\sum_{k=1}^{N-1} v_{k}\right)^{s}
$$

Combining the two expressions displayed above yields $H_{V}=\frac{N P^{2}}{2}+\tilde{H}_{V}$, where $\tilde{H}_{V}$ only depends on $(v, u)$ and is given by

$$
\begin{aligned}
\tilde{H}_{V}= & \frac{1}{2}\left(u_{1}^{2}+\sum_{l=1}^{N-2}\left(u_{l+1}-u_{l}\right)^{2}+u_{N-1}^{2}\right)+\frac{1}{2}\left(\sum_{k=1}^{N-1} v_{k}^{2}+\left(\sum_{k=1}^{N-1} v_{k}\right)^{2}\right) \\
& +\frac{\alpha}{3 !}\left(\sum_{k=1}^{N-1} v_{k}^{3}-\left(\sum_{k=1}^{N-1} v_{k}\right)^{3}\right)+\frac{\beta}{4 !}\left(\sum_{k=1}^{N-1} v_{k}^{4}+\left(\sum_{k=1}^{N-1} v_{k}\right)^{4}\right)+O\left(v^{5}\right) .
\end{aligned}
$$


Note that for any values of $\alpha$ and $\beta$, the point $(v, u)=(0,0)$ is a critical point of the Hamiltonian $\tilde{H}_{V}$.

To compute the Birkhoff normal form of $\tilde{H}_{V}$ up to order 2 near the fixed point $(v, u)=(0,0)$, we take the expansion (90) as a starting point and use the linearization of the Birkhoff map at $(v, u)=(0,0)\left(\mathrm{cf}\right.$. [7]) to define new coordinates $\left(\xi_{k}, \eta_{k}\right)_{1 \leq k \leq N-1}$. The following lemma gives an independent proof of the fact that this linear map, defined by (14)-(17), is canonical.

Lemma B.1. The linear transformation $z \rightarrow \mathbb{R}^{2 N-2}, \zeta \mapsto(v, u)$, as defined by (14)-(17), is a canonical isomorphism.

Proof. First let us show

$$
\begin{gathered}
\left\{v_{l}(\zeta), u_{m}(\zeta)\right\}=i \delta_{l m}, \\
\left\{v_{l}(\zeta), v_{m}(\zeta)=0\right. \\
\left\{u_{l}(\zeta), u_{m}(\zeta)\right\}=0
\end{gathered}
$$

for any $1 \leq l, m \leq N-1$. Since $(v, u)$ are canonical coordinates on $\mathbb{R}^{2 N-2}$, the proof of (91) amounts to showing that

$$
\sum_{k=1}^{N-1}\left(\frac{\partial v_{l}}{\partial \zeta_{k}} \frac{\partial u_{m}}{\partial \zeta_{-k}}-\frac{\partial v_{l}}{\partial \zeta_{-k}} \frac{\partial u_{m}}{\partial \zeta_{k}}\right)=i \delta_{l m}
$$

for any $1 \leq l, m \leq N-1$. It follows from (14)-(17) that for any $1 \leq k \leq N-1$,

$$
\begin{aligned}
& \frac{\partial v_{l}}{\partial \zeta_{k}}=\frac{\lambda_{k}}{\sqrt{N}} e^{\pi i(2 l-1) k / N}, \quad \frac{\partial v_{l}}{\partial \zeta_{-k}}=\frac{\lambda_{k}}{\sqrt{N}} e^{-\pi i(2 l-1) k / N} \\
& \frac{\partial u_{m}}{\partial \zeta_{k}}=\frac{\lambda_{k}}{\sqrt{N}} \sum_{j=0}^{m-1} e^{2 \pi i j k / N}, \quad \frac{\partial u_{m}}{\partial \zeta_{-k}}=\frac{\lambda_{k}}{\sqrt{N}} \sum_{j=0}^{m-1} e^{-2 \pi i j k / N} .
\end{aligned}
$$

Hence

$$
\begin{aligned}
& \frac{\partial v_{l}}{\partial \zeta_{k}} \frac{\partial u_{m}}{\partial \zeta_{-k}}-\frac{\partial v_{l}}{\partial \zeta_{-k}} \frac{\partial u_{m}}{\partial \zeta_{k}} \\
& =\frac{\lambda_{k}^{2}}{N}\left(e^{\pi i(2 l-1) k / N} \sum_{j=0}^{m-1} e^{-2 \pi i j k / N}-e^{-\pi i(2 l-1) k / N} \sum_{j=0}^{m-1} e^{2 \pi i j k / N}\right) \\
& =\frac{\lambda_{k}^{2}}{N} \sum_{j=0}^{m-1}\left(e^{\frac{\pi i k}{N}(2 l-2 j-1)}-e^{\frac{\pi i k}{N}(2 j-2 l+1)}\right) \\
& =\frac{2 i}{N} \sin \frac{k \pi}{N} \sum_{j=0}^{m-1} \sin \left(\frac{k \pi}{N}(2(l-j)-1)\right) \\
& \quad=\frac{i}{N} \sum_{j=0}^{m-1}\left(\cos \frac{2 k \pi(1-(l-j))}{N}-\cos \frac{2 k \pi(l-j)}{N}\right)
\end{aligned}
$$


where for the latter identity we used that $2 \sin x \sin y=\cos (x-y)-\cos (x+y)$. Taking the sum over $k$ and changing the order of summation then leads to

$$
\begin{aligned}
\sum_{k=1}^{N-1}\left(\frac{\partial v_{l}}{\partial \zeta_{k}} \frac{\partial u_{m}}{\partial \zeta_{-k}}-\frac{\partial v_{l}}{\partial \zeta_{-k}} \frac{\partial u_{m}}{\partial \zeta_{k}}\right) & =\frac{i}{N} \sum_{j=0}^{m-1} \sum_{k=1}^{N-1}\left(\cos \frac{2 k \pi(1-(l-j))}{N}-\cos \frac{2 k \pi(l-j)}{N}\right) \\
& =\frac{i}{N} \sum_{j=0}^{m-1} N\left(\delta_{l-j, 1}-\delta_{l-j, 0}\right) \\
& =i \sum_{j=0}^{m-1}\left(\delta_{l, j+1}-\delta_{l, j}\right)=i\left(\delta_{l m}-\delta_{l 0}\right)=i \delta_{l m},
\end{aligned}
$$

as claimed. To prove (92) and (93) one argues in a similar way. From (91)-(93) it immediately follows that the linear map $\xi \mapsto(v, u)$ is a canonical isomorphism.

We now compute $\tilde{H}_{V}$ in terms of the new variables $\zeta$. Write $\tilde{H}_{V}$ as $\tilde{H}_{V}=H_{u}+H_{v}$, where $H_{u}$ and $H_{v}$ denote the $u$ - and $v$-dependent parts of (90), respectively. We compute $H_{u}(\zeta)$ and $H_{v}(\zeta)$ separately. To obtain $H_{u}(\zeta)$, we substitute (14)-(16) into the expression $\frac{1}{2}\left(u_{1}^{2}+\sum_{l=1}^{N-2}\left(u_{l+1}-u_{l}\right)^{2}+u_{N-1}^{2}\right)$ and get

$$
\begin{aligned}
H_{u}(\zeta) & =\frac{1}{2 N} \sum_{l=0}^{N-1}\left(\sum_{1 \leq|k| \leq N-1} \lambda_{k} e^{2 \pi i l k / N} \zeta_{k}\right)^{2} \\
& =\frac{1}{2 N} \sum_{1 \leq|k|,\left|k^{\prime}\right| \leq N-1} \lambda_{k} \lambda_{k^{\prime}}\left(\sum_{l=0}^{N-1} e^{2 \pi i l\left(k+k^{\prime}\right) / N}\right) \zeta_{k} \zeta_{k^{\prime}}
\end{aligned}
$$

Using again that $\sum_{l=0}^{N-1} e^{2 \pi i l k / N}=N \delta_{k 0}$ and $\lambda_{k}=\lambda_{-k}$ for any $1 \leq|k| \leq N-1$, one obtains

$$
H_{u}(\zeta)=\sum_{k=1}^{N-1} \lambda_{k}^{2} \zeta_{k} \zeta_{-k}
$$

Before computing $H_{v}(\zeta)$, we simplify its expansion in terms of the variables $\left(v_{k}\right)_{1 \leq k \leq N-1}$. Define $v_{0}$ by the expression on the right-hand side of (17) evaluated at $l=0$. Note that

$$
\sum_{l=0}^{N-1} v_{l}=\frac{1}{\sqrt{N}} \sum_{1 \leq|k| \leq N-1} \lambda_{k} \zeta_{k} e^{-i \pi k / N}\left(\sum_{l=0}^{N-1} e^{2 \pi i l k / N}\right)=0
$$

Hence $\sum_{l=1}^{N-1} v_{l}=-v_{0}$ and therefore

$$
H_{v}=\sum_{l=0}^{N-1}\left(\frac{1}{2} v_{l}^{2}+\frac{\alpha}{3 !} v_{l}^{3}+\frac{\beta}{4 !} v_{l}^{4}\right)+O\left(|v|^{5}\right) .
$$


Substituting the expression (17) for $v_{l}$ in the quadratic term in the expansion (94), we get

$$
\begin{aligned}
\frac{1}{2} \sum_{l=0}^{N-1} v_{l}^{2} & =\frac{1}{2 N} \sum_{1 \leq|k|,\left|k^{\prime}\right| \leq N-1} \lambda_{k} \lambda_{k^{\prime}}\left(\sum_{l=0}^{N-1} e^{2 \pi i l\left(k+k^{\prime}\right) / N}\right) e^{-i \pi\left(k+k^{\prime}\right) / N} \zeta_{k} \zeta_{k^{\prime}} \\
& =\sum_{k=1}^{N-1} \lambda_{k}^{2} \zeta_{k} \zeta_{-k},
\end{aligned}
$$

where we again used that $\lambda_{k}=\lambda_{-k}$ and $\sum_{l=0}^{N-1} e^{2 \pi i l k / N}=N \delta_{k 0}$ for any $0 \leq|k| \leq N-1$.

The terms of third and fourth order in $H_{v}$ are treated similarly. Combining the above computations leads to the claimed formula

$$
\tilde{H}_{V}(\zeta)=G_{2}+\alpha G_{3}+\beta G_{4}+O\left(\zeta^{5}\right)
$$

with $G_{2}, G_{3}$, and $G_{4}$ given by (18), (19), and (20), respectively.

Acknowledgement. It is a great pleasure to thank Yves Colin de Verdière and Percy Deift for valuable comments. We also would like to thank the referee for his suggestions of how to improve the exposition of our paper.

\section{References}

1. Bambusi, D., Ponno, A.: Korteweg-de Vries equation and energy sharing in Fermi-PastaUlam. CHAOS 15, 015107 (2005)

2. Bambusi, D., Ponno, A.: On Metastability in FPU. Commun. Math. Phys. 264, 539-561 (2006)

3. Berman, G.P., Izrailev, F.M.: The Fermi-Pasta-Ulam problem: 50 years of progress. CHAOS 15(1), 015104.1-015104.18 (2005)

4. Broer, H.W.: KAM theory: the legacy of Kolmogorov's 1954 paper. Bull. AMS (New Series) 41(4), 507-521 (2004)

5. Fermi, E., Pasta, J., Ulam, S.: Studies of non linear problems. Los Alamos Rpt. LA-1940 (1955). In: Collected Papers of Enrico Fermi. Chicago, IL: University of Chicago Press, 1965, Volume II, Theory, Methods and Applications, (2nd ed., New York: Marcel Dekker, 2000), pp. 978-988

6. Henrici, A., Kappeler, T.: Global Birkhoff coordinates for the periodic Toda lattice. Preprint, 2006

7. Henrici, A., Kappeler, T.: Birkhoff normal form for the periodic Toda lattice. http://arxiv.org/list/nlin.SI/ 0609045, 2006, to appear in Contemp. Math.

8. Henrici, A., Kappeler, T.: Resonant normal form for even periodic FPU chains. arXiv: 0709.2624 [nlin.SI]

9. Kappeler, T., Pöschel, J.: $K d V \& K A M$. Ergebnisse der Mathematik, 3. Folge, 45. Berlin: Springer, 2003

10. Nishida, T.: A note on an existence of conditionally periodic oscillation in a one-dimensional lattice. Mem. Fac. Engrg. Kyoto Univ. 33, 27-34 (1971)

11. Pöschel, J.: Integrability of Hamiltonian Systems on Cantor Sets. Comm. Pure Appl. Math. 35, 653-695 (1982)

12. Pöschel, J.: On Nekhoroshev's Estimate at an Elliptic Equilibrium. Int. Math. Res. Not. 4, 203-215 (1999)

13. Rink, B.: Symmetry and resonance in periodic FPU chains. Commun. Math. Phys. 218, 665-685 (2001)

14. Rink, B.: Direction reversing travelling waves in the Fermi-Pasta-Ulam chain. J. Nonlinear Science 12, 479-504 (2002)

15. Rink, B.: Proof of Nishida's conjecture on anharmonic lattices. Commun. Math. Phys. 261, 613-627 (2006)

16. Toda, M.: Theory of Nonlinear Lattices, 2nd enl. ed., Springer Series in Solid-State Sciences 20. Berlin: Springer, 1989

17. Van der Waerden, B.L.: Algebra I. Heidelberger Taschenbücher. Berlin: Springer, 1966

18. Weissert, T.P.: The genesis of simulation in dynamics: pursuing the Fermi-Pasta-Ulam problem. New York: Springer, 1997 